\title{
No interactions for a collection of Weyl gravitons intermediated by a scalar field
}

\author{
C. Bizdadea, E. M. Cioroianu ${ }^{\dagger}$ A. C. Lungu ${ }^{\ddagger}$ \\ Faculty of Physics, University of Craiova \\ 13 A. I. Cuza Str., Craiova 200585, Romania
}

December 1, 2018

\begin{abstract}
The cross-couplings among several Weyl gravitons (described in the free limit by a sum of linearized Weyl actions) in the presence of a scalar field are studied with the help of the deformation theory based on local BRST cohomology. Under the hypotheses of locality, smoothness of the interactions in the coupling constant, Poincaré invariance, (background) Lorentz invariance, and the preservation of the number of derivatives on each field, together with the supplementary assumption that the internal metric defined by the sum of Weyl lagrangians is positively defined, we prove that there are no consistent cross-interactions among different Weyl gravitons in the presence of a scalar field. The couplings of a single Weyl graviton to a scalar field are also discussed.
\end{abstract}

PACS number: 11.10.Ef

\section{Introduction}

The study of Weyl gravitons is important in view of the remarkable properties of conformal supergravity [1], as well as by the renewed interest in Weyl

*e-mail address: bizdadea@central.ucv.ro

$\dagger$ e-mail address: manache@central.ucv.ro

‡e-mail address: ac_lungu@yahoo.com 
gravity [2] in connection with the ADS/CFT correspondence. Recently, the impossibility of direct cross-interactions among several Weyl gravitons under certain assumptions has been proved in [3] by means of a cohomological approach based on the lagrangian BRST symmetry [4, 15, 6, 7, 8, 9, 10].

The purpose of this paper is to analyze the indirect cross-couplings among several Weyl gravitons (described in the free limit by a sum of linearized Weyl actions) in the presence of a scalar field. Thus, under the hypotheses of locality, smoothness of the interactions in the coupling constant, Poincaré invariance, (background) Lorentz invariance, and the preservation of the number of derivatives on each field, together with the supplementary assumption that the internal metric defined by the sum of Weyl lagrangians is positively defined, we prove that there are no consistent cross-interactions among different Weyl gravitons in the presence of a scalar field. Our result is obtained in the context of the deformation technique [11] combined with the local BRST cohomology [12].

In order to make the presentation as clear as possible, we initially consider the case of the couplings between a single Weyl field and a scalar field, and compute the interaction terms to order two in the coupling constant. In this manner we obtain that the first two orders of the interacting lagrangian resulting from our setting originate in the development of the full interacting lagrangian (in four spacetime dimensions)

$$
\mathcal{L}^{(\mathrm{int})}=\sqrt{-g}\left[\frac{1}{2}\left(g^{\mu \nu}\left(\partial_{\mu} \phi\right) \partial_{\nu} \phi+\frac{1}{6} \phi^{2} R\right)+\lambda \kappa \phi^{4}\right],
$$

where $g_{\mu \nu}$ is the full metric, $g^{\mu \nu}$ denotes its inverse, $R$ represents the full scalar curvature, $\kappa$ is an arbitrary real constant, and $\lambda$ is the coupling constant. The term $\lambda \kappa \phi^{4}$ is usually omitted in the literature. It appears for instance in the partial gauge-fixing procedure with $\phi=1$ [13] that leads from $\mathcal{L}^{\text {(int) }}$ to the standard Einstein-Hilbert action with a cosmological term. This term is consistent with the gauge symmetries of the lagrangian $\mathcal{L}^{(\mathrm{W})}+\mathcal{L}^{(\mathrm{int})}$, where $\mathcal{L}^{(\mathrm{W})}$ is the full Weyl lagrangian. Based on this result, we begin with a finite sum of linearized Weyl actions and a scalar field, and prove that there are no consistent cross-interactions between different Weyl gravitons in the presence of a scalar field under the hypotheses explained in the above.

This paper is organized in seven sections. In Section 2 we construct the BRST symmetry of a free model with a single Weyl field and a scalar field. Section 3 briefly addresses the deformation procedure based on BRST symmetry. In Section 4 we compute the first two orders of the interactions 
between one Weyl graviton and a scalar field. In Section 5 we analyze the deformed theory obtained in the previous section. Section 6 is devoted to the proof of the fact that there are no consistent cross-interactions among different Weyl gravitons in the presence of a scalar field. Section 7 exposes the main conclusions of the paper. The paper also contains one appendix section, in which a statement mentioned in the body of the paper is proved.

\section{Free model: lagrangian formulation and BRST symmetry}

Our starting point is represented by a free lagrangian action, written as the sum between the linearized Weyl gravity action and the action for a massive real scalar field

$$
\begin{aligned}
S_{0}^{\mathrm{L}}\left[h_{\mu \nu}, \phi\right] & =\frac{1}{2} \int d^{4} x\left[\mathcal{W}_{\mu \nu \alpha \beta} \mathcal{W}^{\mu \nu \alpha \beta}+\left(\partial_{\mu} \phi\right)\left(\partial^{\mu} \phi\right)-m^{2} \phi^{2}\right] \equiv \\
& \equiv \int d^{4} x\left(\mathcal{L}_{0}^{(\mathrm{W})}+\mathcal{L}_{0}^{(\phi)}\right),
\end{aligned}
$$

where $\mathcal{W}_{\mu \nu \alpha \beta}$ is the linearized Weyl tensor in four spacetime dimensions, given in terms of the linearized Riemann tensor $\mathcal{R}_{\mu \nu \alpha \beta}$ and of its traces by

$$
\mathcal{W}_{\mu \nu \alpha \beta}=\mathcal{R}_{\mu \nu \alpha \beta}-\frac{1}{2}\left(\sigma_{\mu[\alpha} \mathcal{R}_{\beta] \nu}-\sigma_{\nu[\alpha} \mathcal{R}_{\beta] \mu}\right)+\frac{1}{6} \mathcal{R} \sigma_{\mu[\alpha} \sigma_{\beta] \nu}
$$

Throughout the paper we work with the flat metric of 'mostly plus' signature $\sigma_{\mu \nu}=(-+++)$. The notation $[\mu \cdots \nu]$ signifies antisymmetrization with respect to all indices between brackets without normalization factors (i.e., the independent terms appear only once and are not multiplied by overall numerical factors). The linearized Riemann tensor is expressed by

$$
\mathcal{R}_{\mu \nu \alpha \beta}=\frac{1}{2}\left(\partial_{\mu} \partial_{\beta} h_{\nu \alpha}+\partial_{\nu} \partial_{\alpha} h_{\mu \beta}-\partial_{\nu} \partial_{\beta} h_{\mu \alpha}-\partial_{\mu} \partial_{\alpha} h_{\nu \beta}\right),
$$

while its simple and respectively double traces read as

$$
\mathcal{R}_{\mu \nu}=\sigma^{\alpha \beta} \mathcal{R}_{\mu \alpha \nu \beta}, \quad \mathcal{R}=\sigma^{\mu \nu} \mathcal{R}_{\mu \nu} .
$$

The linearized Weyl tensor can be expressed in terms of the symmetric tensor $\mathcal{K}_{\mu \nu}$ like

$$
\mathcal{W}_{\mu \nu \alpha \beta}=\mathcal{R}_{\mu \nu \alpha \beta}-\left(\sigma_{\mu[\alpha} \mathcal{K}_{\beta] \nu}-\sigma_{\nu[\alpha} \mathcal{K}_{\beta] \mu}\right)
$$


where

$$
\mathcal{K}_{\mu \nu}=\frac{1}{2}\left(\mathcal{R}_{\mu \nu}-\frac{1}{6} \sigma_{\mu \nu} \mathcal{R}\right), \quad \mathcal{K}=\frac{1}{6} \mathcal{R}
$$

The theory described by the action (11) possesses an irreducible and abelian generating set of gauge transformations

$$
\delta_{\epsilon} h_{\mu \nu}=\partial_{(\mu} \epsilon_{\nu)}+2 \sigma_{\mu \nu} \epsilon, \quad \delta_{\epsilon} \phi=0,
$$

where the gauge parameters $\epsilon_{\mu}$ and $\epsilon$ are bosonic functions. The scalar gauge parameter $\epsilon$ is responsible for the so-called conformal invariance of Weyl theory, while $\epsilon_{\mu}$ appear also in the Pauli-Fierz theory and will be called 'Pauli-Fierz' gauge parameters. The notation $(\mu \nu)$ signifies symmetry with respect to the indices between parentheses without the factor $1 / 2$.

In order to construct the BRST symmetry for the model under study, we introduce the fermionic ghosts $\eta_{\mu}$ and $\xi$ respectively associated with the gauge parameters $\epsilon_{\mu}$ and $\epsilon$. The antifield spectrum is organized into the antifields $\left\{h^{* \mu \nu}, \phi^{*}\right\}$ (of the original fields $\left\{h_{\mu \nu}, \phi\right\}$ ) and $\left\{\eta^{* \mu}, \xi^{*}\right\}$ (of the ghosts $\left.\left\{\eta_{\mu}, \xi\right\}\right)$, with the statistics of the antifields opposite to that of the associated fields/ghosts.

Since the gauge generators of the free theory are field independent, it follows that the BRST differential simply reduces to

$$
s=\delta+\gamma
$$

where $\delta$ represents the Koszul-Tate differential, graded by the antighost number agh $(\operatorname{agh}(\delta)=-1)$, and $\gamma$ stands for the exterior derivative along the gauge orbits, whose degree is named pure ghost number pgh $(\operatorname{pgh}(\gamma)=1)$. These two degrees do not interfere $(\operatorname{pgh}(\delta)=0$, agh $(\gamma)=0)$. The overall degree that grades the BRST complex is known as the ghost number gh and is defined like the difference between the pure ghost number and the antighost number, such that $\mathrm{gh}(\delta)=\mathrm{gh}(\gamma)=1$. If we denote by

$$
\Phi^{\alpha_{0}}=\left(h_{\mu \nu}, \phi\right), \quad \eta^{\alpha_{1}}=\left(\eta_{\mu}, \xi\right)
$$

the fields and ghosts of the free theory $\left(\eta_{\mu}\right.$ will be called the 'Pauli-Fierz' ghosts), and by

$$
\Phi_{\alpha_{0}}^{*}=\left(h^{* \mu \nu}, \phi^{*}\right), \quad \eta_{\alpha_{1}}^{*}=\left(\eta^{* \mu}, \xi^{*}\right)
$$


the corresponding antifields, then, according to the standard rules of the BRST formalism, the corresponding degrees of the generators from the BRST complex are valued like

$$
\begin{aligned}
\operatorname{agh}\left(\Phi^{\alpha_{0}}\right)=0, & \operatorname{agh}\left(\eta^{\alpha_{1}}\right)=0, \\
\operatorname{agh}\left(\Phi_{\alpha_{0}}^{*}\right)=1, & \operatorname{agh}\left(\eta_{\alpha_{1}}^{*}\right)=2, \\
\operatorname{pgh}\left(\Phi^{\alpha_{0}}\right)=0, & \operatorname{pgh}\left(\eta^{\alpha_{1}}\right)=1, \\
\operatorname{pgh}\left(\Phi_{\alpha_{0}}^{*}\right)=0, & \operatorname{pgh}\left(\eta_{\alpha_{1}}^{*}\right)=0 .
\end{aligned}
$$

The actions of the differentials $\delta$ and $\gamma$ on the generators (91)-(10) from the BRST complex are given by

$$
\begin{aligned}
\delta h^{* \mu \nu} & =2 \partial_{\alpha} \partial_{\beta} \mathcal{W}^{\mu \alpha \nu \beta}, \quad \delta \phi^{*}=\left(\square+m^{2}\right) \phi \\
\delta \eta^{* \mu} & =-2 \partial_{\nu} h^{* \mu \nu}, \quad \delta \xi^{*}=2 h^{*}, \\
\delta \Phi^{\alpha_{0}} & =0, \quad \delta \eta^{\alpha_{1}}=0, \\
\gamma \Phi_{\alpha_{0}}^{*} & =0, \quad \gamma \eta_{\alpha_{1}}^{*}=0, \quad \gamma \phi=0, \\
\gamma h_{\mu \nu} & =\partial_{(\mu} \eta_{\nu)}+2 \sigma_{\mu \nu} \xi, \quad \gamma \xi=0,
\end{aligned}
$$

being understood that both operators act like right derivations. The notation $h^{*}$ signifies the trace of $h^{* \mu \nu}, h^{*}=\sigma_{\mu \nu} h^{* \mu \nu}$. The BRST differential is known to have a canonical action in a structure named antibracket and denoted by the symbol $(),(s \cdot=(\cdot, \bar{S}))$, which is obtained by setting the fields and ghosts respectively conjugated to the corresponding antifields. The generator of the BRST symmetry is a bosonic functional, of ghost number zero, which is solution to the classical master equation $(\bar{S}, \bar{S})=0$. In our case the solution to the master equation reads as

$$
\bar{S}=S_{0}^{\mathrm{L}}\left[h_{\mu \nu}, \phi\right]+\int d^{4} x h^{* \mu \nu}\left(\partial_{(\mu} \eta_{\nu)}+2 \sigma_{\mu \nu} \xi\right) .
$$

\section{Deformation of the solution to the master equation: a brief review}

We begin with a "free" gauge theory, described by a lagrangian action $S_{0}^{\mathrm{L}}\left[\Phi^{\alpha_{0}}\right]$, invariant under some gauge transformations

$$
\delta_{\epsilon} \Phi^{\alpha_{0}}=Z_{\alpha_{1}}^{\alpha_{0}} \epsilon^{\alpha_{1}}, \quad \frac{\delta S_{0}^{\mathrm{L}}}{\delta \Phi^{\alpha_{0}}} Z_{\alpha_{1}}^{\alpha_{0}}=0
$$


and consider the problem of constructing consistent interactions among the fields $\Phi^{\alpha_{0}}$ such that the couplings preserve the field spectrum and the original number of gauge symmetries. This matter is addressed by means of reformulating the problem of constructing consistent interactions as a deformation problem of the solution to the master equation corresponding to the "free" theory [11]. Such a reformulation is possible due to the fact that the solution to the master equation contains all the information on the gauge structure of the theory. If an interacting gauge theory can be consistently constructed, then the solution $\bar{S}$ to the master equation associated with the "free" theory, $(\bar{S}, \bar{S})=0$, can be deformed into a solution $S$

$$
\begin{aligned}
\bar{S} \rightarrow S & =\bar{S}+\lambda S_{1}+\lambda^{2} S_{2}+\cdots= \\
& =\bar{S}+\lambda \int d^{D} x a+\lambda^{2} \int d^{D} x b+\cdots
\end{aligned}
$$

of the master equation for the deformed theory

$$
(S, S)=0
$$

such that both the ghost and antifield spectra of the initial theory are preserved. The equation (24) splits, according to the various orders in the coupling constant (deformation parameter) $\lambda$, into

$$
\begin{aligned}
(\bar{S}, \bar{S}) & =0 \\
2\left(S_{1}, \bar{S}\right) & =0 \\
2\left(S_{2}, \bar{S}\right)+\left(S_{1}, S_{1}\right) & =0 \\
\left(S_{3}, \bar{S}\right)+\left(S_{1}, S_{2}\right) & =0
\end{aligned}
$$

The equation (25) is fulfilled by hypothesis. The next one requires that the first-order deformation of the solution to the master equation, $S_{1}$, is a co-cycle of the "free" BRST differential $s, s S_{1}=0$. However, only cohomologically nontrivial solutions to (26) should be taken into account, as the BRST-exact ones can be eliminated by some (in general nonlinear) field redefinitions. This means that $S_{1}$ pertains to the ghost number zero cohomological space of $s, H^{0}(s)$, which is generically nonempty due to its isomorphism to the space of physical observables of the "free" theory. It has been shown (on behalf of the triviality of the antibracket map in the cohomology of the 
BRST differential) that there are no obstructions in finding solutions to the remaining equations $((27)-(28)$, etc. $)$. However, the resulting interactions may be nonlocal, and there might even appear obstructions if one insists on their locality. The analysis of these obstructions can be done with the help of cohomological techniques.

\section{Consistent interactions between the Weyl graviton and the real scalar field}

The aim of this section is to investigate the cross-couplings that can be introduced between a single Weyl graviton and a scalar field. This matter is addressed in the context of the antifield-BRST deformation procedure described in the above and relies on computing the solutions to the equations (26) -(28), etc., with the help of the BRST cohomology of the free theory. The interactions are obtained under the following (reasonable) assumptions: smoothness in the deformation parameter, locality, (background) Lorentz invariance, Poincaré invariance, and preservation of the number of derivatives on each field. 'Smoothness of the deformations' refers to the fact that the deformed solution to the master equation (23) is smooth in the coupling constant $\lambda$ and reduces to the original solution (21) in the free limit $\lambda=0$. The requirement that the interacting theory is Poincaré invariant means that one does not allow an explicit dependence of the deformed solution to the master

equation on the spacetime coordinates. The conservation of the number of derivatives on each field with respect to the free theory means here that the following two requirements are simultaneously satisfied: (i) the derivative order of the equations of motion on each field is the same for the free and respectively for the interacting theory; (ii) the maximum number of derivatives in the interaction vertices is equal to four, i.e. the maximum number of derivatives from the free lagrangian. Conditions of this type are frequently imposed in the literature at the level of constructing interacting theories; for instance, see the case of cross-interactions for a collection of Pauli-Fierz fields [14], the couplings between the Pauli-Fierz and the massless RaritaSchwinger fields [15], or the direct cross-interactions for a collection of Weyl gravitons [3]. 


\subsection{Standard material: $H(\gamma)$ and $H(\delta \mid d)$}

The equation (26), which we have seen that controls the first-order deformation, takes the local form

$$
s a=\partial_{\mu} m^{\mu}, \quad \operatorname{gh}(a)=0, \quad \varepsilon(a)=0,
$$

for some local $m^{\mu}$, and it shows that the nonintegrated density of the firstorder deformation pertains to the local cohomology of the free BRST differential in ghost number zero, $a \in H^{0}(s \mid d)$, where $d$ denotes the exterior spacetime differential. The solution to the equation (29) is unique up to $s$-exact pieces plus divergences

$$
a \rightarrow a+s b+\partial_{\mu} n^{\mu}
$$

where

$$
\operatorname{gh}(b)=-1, \quad \varepsilon(b)=1, \quad \operatorname{gh}\left(n^{\mu}\right)=0, \quad \varepsilon\left(n^{\mu}\right)=0 .
$$

At the same time, if the general solution of (29) is found to be completely trivial, $a=s b+\partial_{\mu} n^{\mu}$, then it can be made to vanish $a=0$.

In order to analyze the equation (29), we develop $a$ according to the antighost number

$$
a=\sum_{i=0}^{I} a_{i}, \quad \operatorname{agh}\left(a_{i}\right)=i, \quad \operatorname{gh}\left(a_{i}\right)=0, \quad \varepsilon\left(a_{i}\right)=0,
$$

and assume, without loss of generality, that the decomposition (31) stops at some finite value of $I$. This can be shown for instance like in Appendix A of [3]. Replacing the decomposition (31) into the equation (29) and projecting it on the various values of the antighost number by means of the splitting (8)), we obtain the tower of equations

$$
\begin{aligned}
\gamma a_{I} & =\partial_{\mu} \stackrel{(I)}{m}^{\mu}, \\
\delta a_{I}+\gamma a_{I-1} & =\partial_{\mu} \stackrel{(I-1)}{m}^{\mu}, \\
\delta a_{i}+\gamma a_{i-1} & =\partial_{\mu} \stackrel{(i-1)}{m}^{\mu}, \quad 1 \leq i \leq I-1,
\end{aligned}
$$

where $\left(\stackrel{(i)}{m}^{\mu}\right)_{i=\overline{0, I}}$ are some local currents with $\operatorname{agh}\left(\stackrel{(i)}{m}^{\mu}\right)=i$. Moreover, according to the general result from [3] in the absence of collection indices, 
the equation (32) can be replaced ${ }^{1}$ in strictly positive antighost numbers by

$$
\gamma a_{I}=0, \quad I>0 .
$$

Due to the second-order nilpotency of $\gamma\left(\gamma^{2}=0\right)$, the solution to the equation (35) is unique up to $\gamma$-exact contributions

$$
a_{I} \rightarrow a_{I}+\gamma b_{I}, \quad \operatorname{agh}\left(b_{I}\right)=I, \quad \operatorname{pgh}\left(b_{I}\right)=I-1, \quad \varepsilon\left(b_{I}\right)=1 .
$$

Meanwhile, if it turns out that $a_{I}$ reduces to $\gamma$-exact terms only, $a_{I}=\gamma b_{I}$, then it can be made to vanish, $a_{I}=0$. In other words, the nontriviality of the first-order deformation $a$ is translated at its highest antighost number component into the requirement that $a_{I} \in H^{I}(\gamma)$, where $H^{I}(\gamma)$ denotes the cohomology of the exterior longitudinal derivative $\gamma$ in pure ghost number equal to $I$. So, in order to solve the equation (29) (equivalent with (35) and (33) -(34) ), we need to compute the cohomology of $\gamma, H(\gamma)$, and, as it will be made clear below, also the local cohomology of $\delta, H(\delta \mid d)$.

Using the results on the cohomology of $\gamma$ in the Weyl sector [3] and the definitions of $\gamma$ acting on the scalar field $\phi$ and on its antifield $\phi^{*}$, we can state that $H(\gamma)$ is generated on the one hand by $\Phi_{\alpha_{0}}^{*}, \eta_{\alpha_{1}}^{*}, \phi$, and $\mathcal{W}_{\mu \nu \alpha \beta}$, as well as by their spacetime derivatives, and, on the other hand, by the ghosts and their first-order derivatives $\eta_{\mu}, \partial_{[\mu} \eta_{\nu]}, \xi$, and $\partial_{\mu} \xi$. So, the most general (and nontrivial), local solution to (35) can be written, up to $\gamma$-exact contributions, as

$$
a_{I}=\alpha_{I}\left([\phi],\left[\mathcal{W}_{\mu \nu \alpha \beta}\right],\left[\Phi_{\alpha_{0}}^{*}\right],\left[\eta_{\alpha_{1}}^{*}\right]\right) \omega^{I}\left(\eta_{\mu}, \partial_{[\mu} \eta_{\nu]}, \xi, \partial_{\mu} \xi\right)
$$

where the notation $f([q])$ means that $f$ depends on $q$ and its derivatives up to a finite order, while $\omega^{I}$ denotes the elements with pure ghost number $I$ of a basis in the space of polynomials in the corresponding ghosts and their firstorder derivatives. The objects $\alpha_{I}$ (obviously nontrivial in $H^{0}(\gamma)$ ) were taken to have a finite antighost number and a bounded number of derivatives, and therefore they are polynomials in the antifields $\Phi_{\alpha_{0}}^{*}$ and $\eta_{\alpha_{1}}^{*}$, in the linearized Weyl tensor $\mathcal{W}_{\mu \nu \alpha \beta}$, in the first-order derivatives of the scalar field $\partial_{\mu} \phi$, as well as in their subsequent derivatives. However, $\alpha_{I}$ may contain infinite, formal series in the undifferentiated scalar field $\phi$. They are required to fulfill the property agh $\left(\alpha_{I}\right)=I$ in order to ensure that the ghost number of

\footnotetext{
${ }^{1}$ This is because the presence of matter fields (in our case a real scalar field) does not modify the general results on $H(\gamma)$ presented in $[3$.
} 
$a_{I}$ is equal to zero. Due to their $\gamma$-closeness, $\gamma \alpha_{I}=0$ and to their (partial) polynomial character, $\alpha_{I}$ will be called "invariant polynomials". In zero antighost number the invariant polynomials are polynomials in the linearized Weyl tensor, in its derivatives, and in the derivatives of the real scalar field, with coefficients that may be infinite series in the undifferentiated real scalar field $\phi$.

Substituting (37) in (33), we obtain that a necessary (but not sufficient) condition for the existence of (nontrivial) solutions $a_{I-1}$ is that the invariant polynomials $\alpha_{I}$ are (nontrivial) objects from the local cohomology of the Koszul-Tate differential $H(\delta \mid d)$ in antighost number $I>0$ and in pure ghost number zero,

$$
\delta \alpha_{I}=\partial_{\mu} \stackrel{(I-1)^{\mu}}{j}, \quad \operatorname{agh}\left(\begin{array}{c}
(I-1)^{\mu} \\
j
\end{array}\right)=I-1, \quad \operatorname{pgh}\left(\begin{array}{c}
(I-1)^{\mu} \\
j
\end{array}\right)=0 .
$$

We recall that the local cohomology $H(\delta \mid d)$ is completely trivial in both strictly positive antighost and pure ghost number (for instance, see Theorem 5.4 in [12] and also [16]). Using the fact that the Cauchy order of the free theory under study is equal to two, together with the general results from [12, according to which the local cohomology of the Koszul-Tate differential in pure ghost number zero is trivial in antighost numbers strictly greater than its Cauchy order, we can state that

$$
H_{J}(\delta \mid d)=0 \quad \text { for all } J>2,
$$

where $H_{J}(\delta \mid d)$ denotes the local cohomology of the Koszul-Tate differential in antighost number $J$ and in pure ghost number equal to zero. It has been shown in detail in [3] (Theorem 5.1) that any invariant polynomial from the Weyl sector that is trivial in $H_{J}(\delta \mid d)$ with $J \geq 2$ can be taken to be trivial also in $H_{J}^{\text {inv }}(\delta \mid d)$. $\left[H_{J}^{\text {inv }}(\delta \mid d)\right.$ denotes the invariant characteristic cohomology in antighost number $J$ (the local cohomology of the Koszul-Tate differential in the space of invariant polynomials).] This property is still valid here since the real scalar field has no gauge invariance of its own, and thus the scalar field sector can intervene nontrivially only in the cohomology of the KoszulTate differential in antighost number one. Thus:

$$
\left(\alpha_{J}=\delta b_{J+1}+\partial_{\mu} \stackrel{(J)}{c}^{\mu}, \operatorname{agh}\left(\alpha_{J}\right)=J \geq 2\right) \Rightarrow \alpha_{J}=\delta \beta_{J+1}+\partial_{\mu} \stackrel{(J)}{\gamma}^{\mu}
$$


with both $\beta_{J+1}$ and $\stackrel{(J)}{\gamma}^{\mu}$ invariant polynomials. The results (39) and (40) yield the conclusion that

$$
H_{J}^{\text {inv }}(\delta \mid d)=0 \quad \text { for all } J>2 .
$$

The antifield of the scalar field brings only trivial contributions to both $H_{J}(\delta \mid d)$ (in pure ghost number zero) and $H_{J}^{\text {inv }}(\delta \mid d)$ for $J \geq 2$, so the results from [3] regarding $H_{2}(\delta \mid d)$ (in pure ghost number zero) and $H_{2}^{\text {inv }}(\delta \mid d)$ remain valid here. Both cohomologies are still spanned by the undifferentiated antifields corresponding to the 'Pauli-Fierz' ghosts

$$
H_{2}(\delta \mid d) \quad \text { and } \quad H_{2}^{\text {inv }}(\delta \mid d):\left(\eta^{* \mu}\right) .
$$

In contrast to the groups $\left(H_{J}(\delta \mid d)\right)_{J \geq 2}$ and $\left(H_{J}^{\text {inv }}(\delta \mid d)\right)_{J \geq 2}$, which are finitedimensional, the cohomology $H_{1}(\delta \mid d)$ in pure ghost number zero, that is related to global symmetries and ordinary conservation laws, is infinitedimensional since the theory is free. Fortunately, it will not be needed in the sequel.

The previous results on $H(\delta \mid d)$ and $H^{\text {inv }}(\delta \mid d)$ in strictly positive antighost numbers are important because they control the obstructions to removing the antifields from the first-order deformation. Based on the formulas (39)-(41), one can successively eliminate all the pieces of antighost number strictly greater that two from the nonintegrated density of the first-order deformation by adding only trivial terms, so one can take, without loss of nontrivial objects, the condition $I \leq 2$ in the decomposition (31). The proof of this statement can be realized like in the subsection 6.1 from [3].

\subsection{First-order deformation}

In the case $I=2$ the nonintegrated density of the first-order deformation (31) becomes

$$
a=a_{0}+a_{1}+a_{2} .
$$

We can further decompose $a$ in a natural manner as

$$
a=a^{(\mathrm{W})}+a^{(\mathrm{int})}+a^{(\phi)},
$$

where $a^{(\mathrm{W})}$ contains only fields/ghosts/antifields from the Weyl sector, $a^{(\mathrm{int})}$ describes the cross-interactions between the Weyl graviton and the scalar field 
(so it effectively mixes both sectors), and $a^{(\phi)}$ involves only the scalar field sector. The component $a^{(\mathrm{W})}$ is completely known [3] and satisfies individually an equation of the type (29). It admits a decomposition similar to (43)

$$
a^{(\mathrm{W})}=a_{0}^{(\mathrm{W})}+a_{1}^{(\mathrm{W})}+a_{2}^{(\mathrm{W})}
$$

where

$$
\begin{aligned}
a_{2}^{(\mathrm{W})}= & \eta^{* \mu}\left(\frac{1}{2} \eta^{\nu} \partial_{[\mu} \eta_{\nu]}+\eta_{\mu} \xi\right)-\xi^{*} \eta_{\mu} \partial^{\mu} \xi \\
a_{1}^{(\mathrm{W})}= & -\frac{1}{2} h^{* \mu \nu} \eta^{\rho}\left(\partial_{(\mu} h_{\nu) \rho}-2 \partial_{\rho} h_{\mu \nu}\right)+ \\
& +2 h^{* \mu \nu} h_{\mu \nu} \xi+\frac{1}{2} h^{* \mu \nu} h_{\rho(\mu} \partial_{\nu)} \eta^{\rho}
\end{aligned}
$$

and $a_{0}^{(\mathrm{W})}$ is the cubic vertex of the Weyl lagrangian2. Since $a^{(\mathrm{int})}$ mixes variables from Weyl and matter sectors, while $a^{(\phi)}$ depends only on the matter field, it follows that these are subject to two separate equations

$$
\begin{aligned}
s a^{(\mathrm{int})} & =\partial_{\mu} m^{(\mathrm{int}) \mu}, \\
s a^{(\phi)} & =\partial_{\mu} m^{(\phi) \mu},
\end{aligned}
$$

for some local $m^{\mu}$ 's. In the sequel we analyze the general solutions to these equations.

Like we mentioned before, the scalar field sector may appear nontrivially only in antighost number one or zero, so one can always take $a_{2}^{(\mathrm{int})}=0$ and hence work with

$$
a^{(\mathrm{int})}=a_{0}^{(\mathrm{int})}+a_{1}^{(\mathrm{int})},
$$

where the components of $a^{(\text {int })}$ are subject to the equations

$$
\begin{aligned}
\gamma a_{1}^{(\mathrm{int})} & =0 \\
\delta a_{1}^{(\mathrm{int})}+\gamma a_{0}^{(\mathrm{int})} & =\partial_{\mu} \stackrel{(0)}{m}^{(\mathrm{int}) \mu} .
\end{aligned}
$$

\footnotetext{
${ }^{2}$ The terms $a_{2}^{(\mathrm{W})}$ and $a_{1}^{(\mathrm{W})}$ given in (46) and (47) differ from the corresponding ones in 3 by a $\gamma$-exact and respectively a $\delta$-exact contribution. However, the difference between our $a_{2}^{(\mathrm{W})}+a_{1}^{(\mathrm{W})}$ and the corresponding sum from 3 is a $s$-exact modulo $d$ quantity. The associated component of antighost number zero, $a_{0}^{(\mathrm{W})}$, is nevertheless the same in both formulations. As a consequence, the object $a^{(\mathrm{W})}$ and the first-order deformation in [3] belong to the same cohomological class from $H^{0}(s \mid d)$.
} 
According to (37) in pure ghost number equal to one, it results that the most general form of $a_{1}^{\text {(int) }}$ as solution to (51) that might provide effective cross-interactions is written like

$$
\begin{aligned}
a_{1}^{(\mathrm{int})}= & \phi^{*}\left(M^{\mu} \partial_{\mu} \xi+\bar{M}^{\mu \nu} \partial_{[\mu} \eta_{\nu]}+\hat{M} \xi+\hat{M}^{\mu} \eta_{\mu}\right)+ \\
& +h^{* \alpha \beta}\left(M_{\alpha \beta}^{\mu} \partial_{\mu} \xi+\bar{M}_{\alpha \beta}^{\mu \nu} \partial_{[\mu} \eta_{\nu]}+\hat{M}_{\alpha \beta} \xi+\hat{M}_{\alpha \beta}^{\mu} \eta_{\mu}\right),
\end{aligned}
$$

where the $M$-like functions may depend on the scalar field, on the linearized Weyl tensor, as well as on their spacetime derivatives, and satisfy obvious 'symmetry' properties. Using the definitions of $\delta$ and $\gamma$, after some computations we obtain that

$$
\delta a_{1}^{(\mathrm{int})}=\gamma b_{0}+\partial_{\mu} j_{0}^{\mu}+c_{0}
$$

where we used the notations

$$
\begin{aligned}
& b_{0}=\sum_{i=1}^{8} b_{0}^{(i)} \text {, } \\
& j^{\mu}=\left[\phi \partial^{\mu} M^{\nu}-M^{\nu} \partial^{\mu} \phi+4 \phi \bar{M}^{\mu \nu}+\sigma^{\mu \nu} \phi \hat{M}-2 M_{\alpha \beta}^{\nu} \partial_{\rho} \mathcal{W}^{\mu \alpha \rho \beta}+\right. \\
& \left.+2 \mathcal{W}^{\mu \alpha \rho \beta}\left(\partial_{\rho} M_{\alpha \beta}^{\nu}+4 \sigma_{\rho \lambda} \bar{M}_{\alpha \beta}^{\lambda \nu}+\delta_{\rho}^{\nu} \hat{M}_{\alpha \beta}\right)\right] \partial_{\nu} \xi+ \\
& +\left[\phi\left(\partial^{\mu} M-\hat{M}^{\mu}\right)-\hat{M} \partial^{\mu} \phi-2 \hat{M}_{\alpha \beta} \partial_{\nu} \mathcal{W}^{\mu \alpha \nu \beta}+\right. \\
& \left.+2 \mathcal{W}^{\mu \alpha \nu \beta}\left(\partial_{\nu} \hat{M}_{\alpha \beta}-\sigma_{\nu \rho} \hat{M}_{\alpha \beta}^{\rho}\right)\right] \xi+ \\
& +\left[\phi \partial^{\mu} \bar{M}^{\rho \lambda}-\bar{M}^{\rho \lambda} \partial^{\mu} \phi+\frac{1}{4} \sigma^{\mu[\rho} \hat{M}^{\lambda]} \phi-2 \bar{M}_{\alpha \beta}^{\rho \lambda} \partial_{\nu} \mathcal{W}^{\mu \alpha \nu \beta}+\right. \\
& \left.+2 \mathcal{W}^{\mu \alpha \nu \beta}\left(\partial_{\nu} \bar{M}_{\alpha \beta}^{\rho \lambda}+\frac{1}{4} \delta_{\nu}^{[\rho} \hat{M}_{\alpha \beta}^{\lambda]}\right)\right] \partial_{[\rho} \eta_{\lambda]}+ \\
& +\left(\phi \partial^{\mu} \hat{M}^{\lambda}-\hat{M}^{\lambda} \partial^{\mu} \phi-2 \hat{M}_{\alpha \beta}^{\lambda} \partial_{\nu} \mathcal{W}^{\mu \alpha \nu \beta}+2 \mathcal{W}^{\mu \alpha \nu \beta} \partial_{\nu} \hat{M}_{\alpha \beta}^{\lambda}\right) \eta_{\lambda},(56 \\
& c_{0}=\left[-\phi\left(\left(\square+m^{2}\right) M^{\lambda}+8 \partial_{\rho} \bar{M}^{\rho \lambda}+2 \hat{M}^{\lambda}+2 \partial^{\lambda} \hat{M}\right)-\right. \\
& -2 \mathcal{W}^{\mu \alpha \nu \beta}\left(\partial_{\mu} \partial_{\nu} M_{\alpha \beta}^{\lambda}+8 \sigma_{\mu \rho} \partial_{\nu} \bar{M}_{\alpha \beta}^{\rho \lambda}+2 \delta_{\nu}^{\lambda} \partial_{\mu} \hat{M}_{\alpha \beta}-\right.
\end{aligned}
$$




$$
\begin{aligned}
& \left.\left.-2 \sigma_{\mu \rho} \delta_{\nu}^{\lambda} \hat{M}_{\alpha \beta}^{\rho}\right)\right] \partial_{\lambda} \xi+ \\
& +\left[-\phi\left(\left(\square+m^{2}\right) \hat{M}+2 \partial_{\lambda} \hat{M}^{\lambda}\right)-\right. \\
& \left.-2 \mathcal{W}^{\mu \alpha \nu \beta}\left(\partial_{\mu} \partial_{\nu} \hat{M}_{\alpha \beta}-2 \sigma_{\mu \rho} \partial_{\nu} \hat{M}_{\alpha \beta}^{\rho}\right)\right] \xi- \\
& -\left[\phi\left(\left(\square+m^{2}\right) \bar{M}^{\rho \lambda}+\frac{1}{2} \partial^{[\rho} \hat{M}^{\lambda]}\right)+\right. \\
& \left.+2 \mathcal{W}^{\mu \alpha \nu \beta}\left(\partial_{\mu} \partial_{\nu} \bar{M}_{\alpha \beta}^{\rho \lambda}+\delta_{\nu}^{\rho} \partial_{\mu} \hat{M}_{\alpha \beta}^{\lambda}\right)\right] \partial_{[\rho} \eta_{\lambda]}- \\
& -\left(\phi\left(\square+m^{2}\right) \hat{M}^{\lambda}+2 \mathcal{W}^{\mu \alpha \nu \beta} \partial_{\mu} \partial_{\nu} \hat{M}_{\alpha \beta}^{\lambda}\right) \eta_{\lambda},
\end{aligned}
$$

as well as

$$
\begin{gathered}
b_{0}^{(1)}=\left(\phi \partial^{\mu} M^{\nu}-M^{\mu} \partial^{\nu} \phi\right) \mathcal{K}_{\mu \nu}, \\
b_{0}^{(2)}=\left(\bar{M}^{\mu \nu} \partial^{\rho} \phi-\phi \partial^{\rho} \bar{M}^{\mu \nu}\right) \partial_{[\mu} h_{\nu] \rho}, \\
b_{0}^{(3)}=\phi \hat{M} \mathcal{K}, \\
b_{0}^{(4)}=\frac{1}{2}\left(\hat{M}^{\mu} \partial^{\nu} \phi-\phi \partial^{\mu} \hat{M}^{\nu}\right) h_{\mu \nu}-\frac{1}{2} \phi \hat{M}^{\mu}\left(\partial^{\nu} h_{\mu \nu}-\partial_{\mu} h\right), \\
b_{0}^{(5)}=-2\left(M_{\alpha \beta}^{\rho} \partial_{\nu} \mathcal{W}^{\mu \alpha \nu \beta}-\mathcal{W}^{\mu \alpha \nu \beta} \partial_{\nu} M_{\alpha \beta}^{\rho}\right) \mathcal{K}_{\mu \rho}, \\
b_{0}^{(6)}=2\left(\bar{M}_{\alpha \beta}^{\rho \lambda} \partial_{\nu} \mathcal{W}^{\mu \alpha \nu \beta}-\mathcal{W}^{\mu \alpha \nu \beta} \partial_{\nu} \bar{M}_{\alpha \beta}^{\rho \lambda}\right) \partial_{[\rho} h_{\lambda] \mu}+ \\
+8 \mathcal{W}^{\mu \alpha \nu \beta} \sigma_{\mu \rho} \bar{M}_{\alpha \beta}^{\rho \lambda} \mathcal{K}_{\lambda \nu}, \\
b_{0}^{(7)}=2 \mathcal{W}^{\mu \alpha \nu \beta} \hat{M}_{\alpha \beta} \mathcal{K}_{\mu \nu}, \\
b_{0}^{(8)}\left(\hat{M}_{\alpha \beta}^{\rho} \partial_{\nu} \mathcal{W}^{\mu \alpha \nu \beta}-\mathcal{W}^{\mu \alpha \nu \beta} \partial_{\nu} \hat{M}_{\alpha \beta}^{\rho}\right) h_{\rho \mu}- \\
-\mathcal{W}^{\mu \alpha \nu \beta} \hat{M}_{\alpha \beta}^{\rho} \partial_{[\mu} h_{\rho] \nu} .
\end{gathered}
$$

According to (52), the terms (58)-(165) give, up to a global factor, some of the pieces from the interacting Lagrangian at order one in the coupling constant. The hypothesis on the conservation of the number of derivatives on each field [conditions (i)-(ii) expressed in the beginning of the section 4] induces further restrictions on the type- $M$ functions, as it will be seen below. The 
second term in (58) outputs a field equation for the scalar field with three derivatives, which disagrees with the condition (i). Therefore, we must set

$$
M^{\mu}=0 .
$$

The pieces (59) satisfy the requirement (i) in relation to the scalar field if the functions $\bar{M}^{\mu \nu}$ depend only on the undifferentiated fields $h_{\alpha \beta}$ and $\phi$. Because $\bar{M}^{\mu \nu}$ are gauge invariant, it results that these functions actually depend on the undifferentiated scalar field. Their antisymmetry property $\bar{M}^{\mu \nu}=-\bar{M}^{\nu \mu}$ prevents the appearance of such functions, and thus we take

$$
\bar{M}^{\mu \nu}=0 \text {. }
$$

The most general form of (60) satisfying (i) is $\hat{M}=M(\phi) u\left(\mathcal{W}^{\mu \nu \alpha \beta} \mathcal{W}_{\mu \nu \alpha \beta}\right)$, with $u$ and $M$ some arbitrary, smooth functions. On the other hand, the condition (ii) prescribes that $\hat{M}$ comprises at most two derivatives (as $\mathcal{K}$ already has two). This finally yields $u=1$ and

$$
\hat{M}=M(\phi) \text {. }
$$

The terms (61) fulfill (i) in relation to the scalar field if the functions $\hat{M}^{\mu}$ depend at most on the first-order derivatives of $\phi$ and $h_{\alpha \beta}$. Since the only gauge-invariant objects constructed out of the graviton field contain at least two derivatives, it follows that $\hat{M}^{\mu}$ can only be written as

$$
\hat{M}^{\mu}=\partial^{\mu} N(\phi),
$$

for some smooth function $N$. Due to the fact that (62)-(63) already produce a field equation for the scalar field with three derivatives, the condition (i) imposes that we must take

$$
M_{\alpha \beta}^{\rho}=0, \quad \bar{M}_{\alpha \beta}^{\rho \lambda}=0 .
$$

Regarding the element (64), it outputs at most a second-order derivative scalar field equation if the functions $\hat{M}_{\alpha \beta}$ are of the type

$$
\hat{M}_{\alpha \beta}=Q(\phi) \mathcal{W}_{\alpha \mu \nu \rho} \mathcal{W}_{\beta}^{\mu \nu \rho} w\left(\mathcal{W}_{\mu \nu \rho \lambda} \mathcal{W}^{\mu \nu \rho \lambda}\right)
$$

where $Q$ and $w$ are smooth functions. If we introduce (71) in (64), then the latter provides an interaction vertex of at least order eight in the derivatives, in disagreement with the condition (ii). Consequently, we have

$$
\hat{M}_{\alpha \beta}=0 \text {. }
$$


Finally, it is easy to see that (65) provide a three-order derivative equation for the scalar field, and therefore we must set

$$
\hat{M}_{\alpha \beta}^{\rho}=0 .
$$

Substituting the results (66) $-(173)$ in (153) and (55) -(57), we arrive at

$$
\begin{gathered}
a_{1}^{(\mathrm{int})}=\phi^{*}\left[M \xi+\left(\partial^{\mu} N\right) \eta_{\mu}\right] \\
b_{0}=\quad \phi M(\phi) \mathcal{K}+\frac{1}{2}\left[\left(\partial^{\mu} N\right) \partial^{\nu} \phi-\phi \partial^{\mu} \partial^{\nu} N\right] h_{\mu \nu}- \\
-\frac{1}{2} \phi\left(\partial^{\mu} N\right)\left(\partial^{\nu} h_{\mu \nu}-\partial_{\mu} h\right), \\
j^{\mu}=\quad \phi M \partial^{\mu} \xi+\left[\phi \partial^{\mu} \partial^{\nu} N-\left(\partial^{\mu} \phi\right) \partial^{\nu} N\right] \eta_{\nu}+ \\
+\left(\phi \partial^{\mu} M-M \partial^{\mu} \phi-\phi \partial^{\mu} N\right) \xi, \\
c_{0}=-2 \phi \partial^{\mu}(M+N) \partial_{\mu} \xi-\phi\left[\left(\square+m^{2}\right) M-2 \square N\right] \xi- \\
-\phi\left[\left(\square+m^{2}\right) \partial^{\mu} N\right] \eta_{\mu} .
\end{gathered}
$$

After some manipulations, the terms (77) can be brought to

$$
c_{0}=\gamma b_{0}^{\prime}+\partial_{\mu} j_{0}^{\prime \mu}+c_{0}^{\prime}
$$

where we employed the notations

$$
\begin{aligned}
j_{0}^{\prime \mu}= & \left(\partial^{\mu} \phi\right)\left(\partial^{\nu} \phi\right) \frac{d N(\phi)}{d \phi} \eta_{\nu}-\left[\frac{1}{2}\left(\partial^{\rho} \phi\right)\left(\partial_{\rho} \phi\right) \frac{d N(\phi)}{d \phi}+\right. \\
& \left.+\phi \square N+m^{2} \sum_{n=2}^{\infty} \frac{(-)^{n}}{n !} \phi^{n} \frac{d^{n-1} N(\phi)}{d \phi^{n-1}}\right] \eta^{\mu}-\bar{M}(\phi) \partial^{\mu} \xi- \\
& -\phi \xi \partial^{\mu}(M+2 N), \\
b_{0}^{\prime}= & \frac{1}{2}\left[-\left(\partial^{\mu} \phi\right) \partial^{\nu} N+\sigma^{\mu \nu}\left(\frac{1}{2}\left(\partial^{\rho} \phi\right) \partial_{\rho} N+\phi \square N\right)\right] h_{\mu \nu}- \\
& -\bar{M}(\phi) \mathcal{K}+\frac{m^{2}}{2}\left(\sum_{n=2}^{\infty} \frac{(-)^{n}}{n !} \phi^{n} \frac{d^{n-1} N(\phi)}{d \phi^{n-1}}\right) h,
\end{aligned}
$$


and

$$
\begin{aligned}
c_{0}^{\prime}= & \frac{1}{2}\left(\partial^{\mu} \phi\right)\left(\partial_{\mu} \phi\right)\left(\partial_{\nu} \phi\right) \frac{d^{2} N(\phi)}{d \phi^{2}} \eta^{\nu}+ \\
& +\left(\partial^{\mu} \phi\right)\left(\partial_{\mu} \phi\right) \frac{d(M(\phi)+N(\phi))}{d \phi} \xi- \\
& -m^{2}\left(\phi M(\phi)+4 \sum_{n=2}^{\infty} \frac{(-)^{n}}{n !} \phi^{n} \frac{d^{n-1} N(\phi)}{d \phi^{n-1}}\right) \xi .
\end{aligned}
$$

In the above $\bar{M}(\phi)$ is defined via the relation $d \bar{M}(\phi) / d \phi=\phi(d M(\phi) / d \phi)$. Inserting (78) in (54), we deduce

$$
\delta a_{1}^{(\mathrm{int})}=\gamma\left(b_{0}+b_{0}^{\prime}\right)+\partial_{\mu}\left(j_{0}^{\mu}+j_{0}^{\prime \mu}\right)+c_{0}^{\prime} .
$$

Because the piece $c_{0}^{\prime}$ is nontrivial in $H(\gamma)$ in pure ghost number one, the existence of $a_{0}^{\text {(int) }}$ as solution to the equation (52) demands that $c_{0}^{\prime}$ must vanish, which further implies the following relations that must be satisfied by the functions $N(\phi)$ and $M(\phi)$ :

$$
\begin{aligned}
\frac{d^{2} N(\phi)}{d \phi^{2}} & =0 \\
\frac{d(M(\phi)+N(\phi))}{d \phi} & =0 \\
m^{2}\left(\phi M(\phi)+4 \sum_{n=2}^{\infty} \frac{(-)^{n}}{n !} \phi^{n} \frac{d^{n-1} N(\phi)}{d \phi^{n-1}}\right) & =0 .
\end{aligned}
$$

From (82) we find that

$$
N(\phi)=c_{1}-k \phi
$$

with $c_{1}$ and $k$ two arbitrary, real constants. Inserting (85) in (83), we have that

$$
M(\phi)=\bar{k}+k \phi
$$

with $\bar{k}$ another arbitrary, real constant. Introducing the solutions (85)-(86) in (84), the last one becomes

$$
-m^{2} k \phi^{2}+m^{2} \bar{k} \phi=0
$$

and leads to

$$
m^{2} k=0, \quad m^{2} \bar{k}=0 .
$$


There are two types of solutions to (88). The former has the form

$$
k=\bar{k}=0, \quad m^{2} \neq 0,
$$

and obviously gives no cross-couplings between the Weyl graviton and the scalar field. For this reason this solution is not interesting, and therefore it will be omitted in the sequel. The latter solution is written like

$$
k \neq 0, \quad \bar{k} \neq 0, \quad m^{2}=0,
$$

and provides the first-order deformation

$$
\begin{gathered}
a_{1}^{\text {(int) }}=\bar{k} \phi^{*} \xi+k \phi^{*}\left[\phi \xi-\left(\partial^{\mu} \phi\right) \eta_{\mu}\right] \\
a_{0}^{(\text {int })}=-\left(\bar{k} \phi+\frac{k}{2} \phi^{2}\right) \mathcal{K}+\frac{k}{2}\left[\left(\partial^{\mu} \phi\right) \partial^{\nu} \phi-\frac{1}{2} \sigma^{\mu \nu}\left(\partial^{\rho} \phi\right) \partial_{\rho} \phi\right] h_{\mu \nu}+\bar{a}_{0}^{\text {(int) }},
\end{gathered}
$$

where $\bar{a}_{0}^{(\text {int })}$ is solution to the 'homogeneous' equation

$$
\gamma \bar{a}_{0}^{(\mathrm{int})}=\partial_{\mu} \bar{m}^{(\mathrm{int}) \mu},
$$

for some local $\bar{m}^{(\mathrm{int}) \mu}$. The solutions to the 'homogeneous' equation correspond to $\bar{a}_{1}^{\text {(int) }}=0$, and thus they do not deform the gauge transformations, but only the interacting lagrangian at order one in the coupling constant. The constant $c_{1}$ appearing in (85) brings no contribution to the first-order deformation and will be set zero in the sequel.

Next, we analyze the equation (93). There are two main types of solutions to this equation. The first type, to be denoted by $\bar{a}_{0}^{\prime(\text { int })}$, corresponds to $\bar{m}^{\text {(int) }) \mu}=0$ and is given by gauge-invariant, nonintegrated densities constructed out of the original fields and their spacetime derivatives, which, according to (37), are of the form

$$
\bar{a}_{0}^{\prime \text { (int) }}=\bar{a}_{0}^{\prime \text { (int) }}\left([\phi],\left[\mathcal{W}_{\mu \nu \alpha \beta}\right]\right),
$$

up to the condition that they effectively describe cross-couplings between the two types of fields and cannot be written in a divergence-like form. The sole possibility that complies with all the hypotheses mentioned in the beginning of this section is

$$
\bar{a}_{0}^{\prime \text { (int) }}=\frac{1}{2} \mathcal{W}_{\mu \nu \rho \lambda} \mathcal{W}^{\mu \nu \rho \lambda} v(\phi),
$$


where $v(\phi)$ is a smooth function of the undifferentiated scalar field. The second kind of solutions, to be denoted by $\bar{a}_{0}^{\prime \prime(\text { int })}$, is associated with $\bar{m}^{\text {(int) } \mu} \neq$ 0 in (93), being understood that we discard the divergence-like quantities and maintain the condition on the conservation of the number of derivatives on each field. At this point it is useful to decompose the exterior derivative along the gauge orbits $\gamma$ like in [3]

$$
\gamma=\gamma_{0}+\gamma_{1}
$$

where $\gamma_{0}$ and $\gamma_{1}$ act nontrivially only on the Weyl field through

$$
\gamma_{0} h_{\mu \nu}=\partial_{(\mu} \eta_{\nu)}, \quad \gamma_{1} h_{\mu \nu}=2 \sigma_{\mu \nu} \xi
$$

The grading associated with this splitting is the number of the ghosts $\xi$ and of their derivatives $\left(\gamma_{1}\right.$ increases this number by one unit, while $\gamma_{0}$ does not affect it). If one plugs this decomposition into the equation (93) corresponding to $\bar{m}^{\text {(int) } \mu} \neq 0$, it becomes equivalent to two equations, namely

$$
\gamma_{0} \bar{a}_{0}^{\prime \prime(\mathrm{int})}=\partial_{\mu} \bar{m}_{0}^{(\mathrm{int}) \mu}, \quad \gamma_{1} \bar{a}_{0}^{\prime \prime(\mathrm{int})}=\partial_{\mu} \bar{m}_{1}^{(\mathrm{int}) \mu} .
$$

As it is shown in Appendix A, the solution to the former equation in (98) reads as

$$
\bar{a}_{0}^{\prime \prime(\mathrm{int})}=\left(c_{1} \mathcal{R}_{\mu \alpha \nu \beta} \mathcal{R}^{\mu \alpha \nu \beta}+c_{2} \mathcal{R}_{\mu \nu} \mathcal{R}^{\mu \nu}+c_{3} \mathcal{R}^{2}\right) \bar{f}(\phi)+\mathcal{R} f(\phi),
$$

with $f(\phi)$ and $\bar{f}(\phi)$ arbitrary, smooth functions of the undifferentiated scalar field, while $c_{1}, c_{2}$, and $c_{3}$ are some arbitrary, real constants. Inserting (99) into the latter equation from (98), we obtain that

$$
\begin{aligned}
& -\bar{f}(\phi)\left[4\left(2 c_{1}+c_{2}\right) \mathcal{R}^{\mu \nu} \partial_{\mu} \partial_{\nu} \xi+2\left(c_{2}+6 c_{3}\right) \mathcal{R} \square \xi\right]- \\
& -6 f(\phi) \square \xi=\partial_{\mu} \bar{m}_{1}^{(\text {int }) \mu} .
\end{aligned}
$$

The left-hand side of (100) reduces to a divergence if

$$
c_{1}=c_{2}=c_{3}=0, \quad f(\phi)=\text { constant. }
$$

The relations (99) and (101) lead to an $\bar{a}_{0}^{\prime \prime(\mathrm{int})}$ that cannot describe crosscouplings, and consequently we must take 3

$$
\bar{a}_{0}^{\prime \prime(\text { int })}=0 .
$$

\footnotetext{
${ }^{3}$ Apparently, a more general solution to the equation (100) would be $c_{1}=-\frac{1}{2} c_{2}, c_{3}=$ $-\frac{1}{6} c_{2}, f(\phi)=$ constant. However, this solution provides a vertex of the type (95), which has already been considered.
} 
Finally, we focus on the solutions to the equation (49). As the scalar field action from (1) has no nontrivial gauge invariance, it follows that $a^{(\phi)}$ can only reduce to its component of antighost number zero

$$
a^{(\phi)} \equiv \bar{a}^{(\phi)}=\bar{a}_{0}^{(\phi)}([\phi]),
$$

which is automatically solution to the equation $s \bar{a}^{(\phi)} \equiv \gamma \bar{a}_{0}^{(\phi)}=0$. It comes from $\bar{a}_{1}^{(\phi)}=0$ and does not deform the gauge transformations (7), but merely modifies the scalar field action. The hypothesis on the conservation of the number of derivatives on each field is translated at the level of $\bar{a}^{(\phi)}$ into

$$
\bar{a}_{0}^{(\phi)}=J(\phi)\left(\partial_{\mu} \phi\right)\left(\partial^{\mu} \phi\right)\left(\partial_{\rho} \phi\right) \partial^{\rho} \phi+F(\phi)\left(\partial_{\rho} \phi\right) \partial^{\rho} \phi+G(\phi),
$$

where $J(\phi), F(\phi)$ and $G(\phi)$ are some arbitrary functions of the undifferentiated scalar field. We can summarize the above results by concluding that the 'interacting' part of the first-order deformation of the solution to the master equation can be written as

$$
\begin{aligned}
S_{1}^{(\mathrm{int})}= & \int d^{4} x\left\{\phi^{*}\left[\bar{k} \xi+k\left(\phi \xi-\left(\partial^{\mu} \phi\right) \eta_{\mu}\right)\right]-\bar{k} \phi \sigma^{\mu \nu} \mathcal{K}_{\mu \nu}-\right. \\
& -\frac{k}{2}\left[\phi^{2} \sigma^{\mu \nu} \mathcal{K}_{\mu \nu}-h_{\mu \nu}\left(\left(\partial^{\mu} \phi\right) \partial^{\nu} \phi-\frac{1}{2} \sigma^{\mu \nu}\left(\partial^{\rho} \phi\right) \partial_{\rho} \phi\right)\right]+ \\
& +\frac{1}{2} \mathcal{W}_{\mu \nu \rho \lambda} \mathcal{W}^{\mu \nu \rho \lambda} v(\phi)+J(\phi)\left(\partial_{\mu} \phi\right)\left(\partial^{\mu} \phi\right)\left(\partial_{\rho} \phi\right) \partial^{\rho} \phi+ \\
& \left.+F(\phi)\left(\partial_{\rho} \phi\right) \partial^{\rho} \phi+G(\phi)\right\}
\end{aligned}
$$

\subsection{Second-order deformation}

So far we have seen that the first-order deformation can be written like the sum between the first-order deformation for the Weyl theory $S_{1}^{(\mathrm{W})}$ (exposed in [3]) and the interacting part $S_{1}^{\text {(int) }}$, given in (105).

In this section we investigate the consistency of the first-order deformation, described by the equation (27). Along the same line as before, we can write the second-order deformation like the sum between the Weyl contribution and the interacting part

$$
S_{2}=S_{2}^{(\mathrm{W})}+S_{2}^{(\mathrm{int})}
$$

The piece $S_{2}^{(\mathrm{W})}$ can be deduced from [3], while $S_{2}^{(\mathrm{int})}$ must satisfy the equation

$$
\frac{1}{2}\left(S_{1}, S_{1}\right)^{(\mathrm{int})}+s S_{2}^{(\mathrm{int})}=0,
$$


where

$$
\left(S_{1}, S_{1}\right)^{(\mathrm{int})}=\left(S_{1}^{(\mathrm{int})}, S_{1}^{(\mathrm{int})}\right)+2\left(S_{1}^{(\mathrm{W})}, S_{1}^{(\mathrm{int})}\right) .
$$

If we denote by $\Lambda^{(\mathrm{int})}$ and $b^{(\mathrm{int})}$ the nonintegrated densities of the functionals $\left(S_{1}, S_{1}\right)^{(\mathrm{int})}$ and respectively $S_{2}^{(\mathrm{int})}$, the local form of (107) becomes

$$
\Lambda^{(\mathrm{int})}=-2 s b^{(\mathrm{int})}+\partial_{\mu} n^{\mu}
$$

with

$$
\operatorname{gh}\left(\Lambda^{(\mathrm{int})}\right)=1, \quad \operatorname{gh}\left(b^{(\mathrm{int})}\right)=0, \quad \operatorname{gh}\left(n^{\mu}\right)=1,
$$

for some local currents $n^{\mu}$. Direct computation shows that $\Lambda^{\text {(int) }}$ decomposes like

$$
\Lambda^{(\mathrm{int})}=\Lambda_{0}^{(\mathrm{int})}+\Lambda_{1}^{(\mathrm{int})}, \quad \operatorname{agh}\left(\Lambda_{I}^{(\mathrm{int})}\right)=I, \quad I=0,1
$$

with

$$
\begin{aligned}
\Lambda_{1}^{(\mathrm{int})}= & -2(1+k)\left[\left(\bar{k} \phi^{*}+k \phi^{*} \phi\right) \eta^{\mu} \partial_{\mu} \xi+k \phi^{*}\left(\partial^{\mu} \phi\right) \eta_{\mu} \xi+\right. \\
& \left.+\frac{k}{2} \phi^{*}\left(\partial^{\mu} \phi\right) \eta^{\nu} \partial_{[\mu} \eta_{\nu]}\right]+\gamma\left(k^{2} \phi^{*}\left(\partial^{\mu} \phi\right) \eta^{\nu} h_{\mu \nu}\right),
\end{aligned}
$$

and

$$
\begin{aligned}
\Lambda_{0}^{(\text {int })=} & -2\left[\bar{k}(\bar{k}+2 k \phi)+k^{2} \phi^{2}\right] \mathcal{K} \xi+2 k\left(h_{\mu \nu}-\frac{1}{2} \sigma_{\mu \nu} h\right) \times \\
& \times\left\{\bar{k}\left(\partial^{\mu} \phi\right) \partial^{\nu} \xi+k\left[\left(\partial^{\mu} \phi\right) \partial^{\nu}(\phi \xi)-\left(\partial^{\mu} \phi\right) \partial^{\nu}\left(\left(\partial^{\rho} \phi\right) \eta_{\rho}\right)\right]+\right. \\
& +2 k(\bar{k}+k \phi) \mathcal{K}\left(\partial^{\rho} \phi\right) \eta_{\rho}+k\left[\left(\partial^{\mu} \phi\right) \partial^{\nu} \phi-\frac{1}{2} \sigma^{\mu \nu}\left(\partial^{\rho} \phi\right) \partial_{\rho} \phi\right] \times \\
& \times\left(-\eta^{\lambda} \partial_{\mu} h_{\nu \lambda}+\eta^{\lambda} \partial_{\lambda} h_{\mu \nu}+2 \xi h_{\mu \nu}+h_{\lambda \mu} \partial_{\nu} \eta^{\lambda}\right)+ \\
& +\frac{1}{6}(2 \bar{k}+k \phi) \phi\left(\partial^{\mu} \partial^{\nu}-\sigma^{\mu \nu} \square\right) \times \\
& \times\left(\eta^{\lambda} \partial_{\mu} h_{\nu \lambda}-\eta^{\lambda} \partial_{\lambda} h_{\mu \nu}-2 \xi h_{\mu \nu}-h_{\lambda \mu} \partial_{\nu} \eta^{\lambda}\right)+ \\
& +2 \bar{k}\left[\left(\frac{d F}{d \phi}\left(\partial_{\rho} \phi\right) \partial^{\rho} \phi+\frac{d G}{d \phi}\right) \xi+2 F\left(\partial_{\rho} \phi\right) \partial^{\rho} \xi\right]+ \\
& +2 k\left[\left(\frac{d F}{d \phi}\left(\partial_{\rho} \phi\right) \partial^{\rho} \phi+\frac{d G}{d \phi}\right)\left(\phi \xi-\left(\partial^{\mu} \phi\right) \eta_{\mu}\right)+\right. \\
& \left.+2 F\left(\partial_{\rho} \phi\right) \partial^{\rho}\left(\phi \xi-\left(\partial^{\mu} \phi\right) \eta_{\mu}\right)\right]+
\end{aligned}
$$




$$
\begin{aligned}
& +2\left[\left(\partial_{\mu} \phi\right)\left(\partial^{\mu} \phi\right)\right]^{2} \xi\left[k\left(\frac{d J(\phi)}{d \phi} \phi+4 J(\phi)\right)+\bar{k} \frac{d J(\phi)}{d \phi}\right]+ \\
& +8\left(\partial_{\mu} \phi\right)\left(\partial^{\mu} \phi\right)\left(\partial_{\nu} \phi\right)\left(\partial^{\nu} \xi\right) J(\phi)(\bar{k}+k \phi)+ \\
& +\gamma\left[\left(-\frac{1}{2} \mathcal{W}^{\mu \nu \rho \lambda} \mathcal{W}_{\mu \nu \rho \lambda} h+4 \mathcal{W}^{\mu \alpha \nu \beta} \sigma^{\rho \lambda} \stackrel{(1)}{\Gamma_{\rho \mu \nu}} \stackrel{(1)}{\Gamma}{ }_{\lambda \alpha \beta}\right) v(\phi)+\right. \\
& +8\left(\mathcal{W}^{\mu \alpha \nu \beta} h_{\mu \nu} \mathcal{K}_{\alpha \beta}+\frac{1}{2} \mathcal{W}^{\mu \alpha \nu \beta} \mathcal{W}_{\mu \rho \nu \beta} h_{\alpha}^{\rho}\right) v(\phi)+ \\
& \left.+k J(\phi)\left(\partial_{\rho} \phi\right)\left(\partial^{\rho} \phi\right)\left(\partial_{\mu} \phi\right)\left(\partial_{\nu} \phi\right)\left(\sigma^{\mu \nu} h-4 h^{\mu \nu}\right)\right]+ \\
& +\mathcal{W}^{\mu \alpha \nu \beta} \mathcal{W}_{\mu \alpha \nu \beta}(\bar{k}+k \phi) \frac{d v(\phi)}{d \phi} \xi- \\
& -(k+1)\left(\mathcal{W}^{\mu \alpha \nu \beta} \mathcal{W}_{\mu \alpha \nu \beta} \frac{d v(\phi)}{d \phi} \partial_{\rho} \phi\right) \eta^{\rho},
\end{aligned}
$$

where

$$
\stackrel{(1)}{\Gamma}_{\mu \nu \rho}=\frac{1}{2}\left(\partial_{\nu} h_{\rho \mu}+\partial_{\rho} h_{\nu \mu}-\partial_{\mu} h_{\nu \rho}\right) .
$$

Since the first-order deformation in the interacting sector starts in antighost number one, we can take, without loss of generality, the corresponding secondorder deformation to start in antighost number two

$$
\begin{aligned}
b^{(\mathrm{int})} & =b_{0}^{(\mathrm{int})}+b_{1}^{(\mathrm{int})}+b_{2}^{(\mathrm{int})}, \quad \operatorname{agh}\left(b_{I}^{(\mathrm{int})}\right)=I, \quad I=0,1,2,(11) \\
n^{\mu} & =n_{0}^{\mu}+n_{1}^{\mu}+n_{2}^{\mu}, \quad \operatorname{agh}\left(n_{I}^{\mu}\right)=I, \quad I=0,1,2 .
\end{aligned}
$$

By projecting the equation (109) on various antighost numbers, we infer the following tower:

$$
\begin{aligned}
\gamma b_{2}^{(\text {int })} & =\partial_{\mu}\left(\frac{1}{2} n_{2}^{\mu}\right), \\
\Lambda_{1}^{(\text {int })} & =-2\left(\delta b_{2}^{(\text {int })}+\gamma b_{1}^{(\text {int) })}+\partial_{\mu} n_{1}^{\mu},\right. \\
\Lambda_{0}^{(\text {int })} & =-2\left(\delta b_{1}^{\text {(int) }}+\gamma b_{0}^{(\text {int })}\right)+\partial_{\mu} n_{0}^{\mu} .
\end{aligned}
$$

As argued in the subsection 4.1, the equation (117) can be always be replaced with

$$
\gamma b_{2}^{(\mathrm{int})}=0 .
$$


If we make the notation

$$
\begin{aligned}
\Gamma= & -2(1+k)\left[\left(\bar{k} \phi^{*}+k \phi^{*} \phi\right) \eta^{\mu} \partial_{\mu} \xi+k \phi^{*}\left(\partial^{\mu} \phi\right) \eta_{\mu} \xi+\right. \\
& \left.+\frac{k}{2} \phi^{*}\left(\partial^{\mu} \phi\right) \eta^{\nu} \partial_{[\mu} \eta_{\nu]}\right]
\end{aligned}
$$

and inspect (112), it results that $\Lambda_{1}^{\text {(int) }}$ can be written like in (118) if

$$
\Gamma=\delta \psi+\gamma \Pi+\partial_{\mu} \rho^{\mu},
$$

for some local nonintegrated quantities $\psi, \Pi$, and $\rho^{\mu}$. By applying $\delta$ on (122), we infer that

$$
\delta \Gamma=\gamma(-\delta \Pi)+\partial_{\mu}\left(\delta \rho^{\mu}\right) .
$$

Let us suppose that (123) holds. Acting now with $\delta$ on (121), we arrive at

$$
\begin{aligned}
\delta \Gamma= & \partial_{\mu}\left\{-2(1+k)\left[\bar{k}\left(\left(\partial^{\mu} \phi\right) \eta^{\nu}-\frac{1}{2} \phi \partial^{[\mu} \eta^{\nu]}\right) \partial_{\nu} \xi+\right.\right. \\
& +k \phi\left(\left(\partial^{\mu} \phi\right) \eta^{\nu}-\frac{1}{4} \phi \partial^{[\mu} \eta^{\nu]}+\frac{1}{2} \phi \xi \sigma^{\mu \nu}\right) \partial_{\nu} \xi- \\
& \left.\left.-k T^{\mu \nu} \eta_{\nu} \xi-\frac{k}{2} T^{\mu \nu} \eta^{\rho} \partial_{[\nu} \eta_{\rho]}\right]\right\}+ \\
& +\gamma\left\{-2(1+k)\left[\bar{k}\left(\partial^{\mu} \phi\right)\left(\eta^{\nu} \mathcal{K}_{\mu \nu}+\frac{1}{2}\left(\partial^{\nu} \xi\right) h_{\mu \nu}\right)-\right.\right. \\
& -\frac{\bar{k}}{2} \phi\left(\partial^{\mu} h_{\mu \nu}-\partial_{\nu} h\right) \partial^{\nu} \xi+k \phi\left(\partial^{\mu} \phi\right)\left(\eta^{\nu} \mathcal{K}_{\mu \nu}+\frac{1}{2}\left(\partial^{\nu} \xi\right) h_{\mu \nu}\right)+ \\
& +\frac{k}{2} \phi^{2}\left(\xi \mathcal{K}+\frac{1}{2}\left(\partial^{\mu} h_{\mu \nu}-\partial_{\nu} h\right) \partial^{\nu} \xi\right)- \\
& -\frac{k}{2} T^{\mu \nu}\left(\xi h_{\mu \nu}+\eta^{\rho} \partial_{\rho} h_{\mu \nu}-\eta^{\rho} \partial_{\mu} h_{\nu \rho}\right)+ \\
& \left.\left.+\frac{k}{4}\left(\partial^{\mu} \phi\right)\left(\partial_{\rho} \phi\right) h^{\rho \nu} \partial_{[\mu} \eta_{\nu]}\right]\right\}
\end{aligned}
$$

where

$$
T^{\mu \nu}=\frac{1}{2} \sigma^{\mu \nu}\left(\partial_{\rho} \phi\right)\left(\partial^{\rho} \phi\right)-\left(\partial^{\mu} \phi\right)\left(\partial^{\nu} \phi\right)
$$

is the stress-energy tensor of the scalar field. Due to the fact that neither of $\eta^{\mu}, \partial^{[\mu} \eta^{\nu]}, \xi$, and $\partial_{\mu} \xi$ are $\delta$-exact, the right-hand side of (124) can be written like in the right-hand side of (123) if

$$
\phi=\delta \Omega,
$$


for some local $\Omega$. Indeed, if $\Omega$ were nonlocal, then (123)-(124) would imply that $\delta \Pi$ is also nonlocal. It is clear that the non-locality of $\delta \Pi$ yields the same with respect to $\Pi$. In this setting, from (112), (118), and (121)-(122) we get that

$$
b_{1}^{\text {(int) }}=-\frac{1}{2}\left(k^{2} \phi^{*}\left(\partial^{\mu} \phi\right) \eta^{\nu} h_{\mu \nu}+\Pi\right),
$$

which indicates that the second-order deformation would contain a nonlocal term, and thus contradicts the hypothesis on the locality of the deformations. We observe that the requirement on $\Omega$ to be a local function is a direct consequence of the required locality of the interactions. On behalf of the second relation in (15) and the last equation from (90) we find that $\phi$ is $\delta$-exact

$$
\phi=\delta\left(\frac{1}{\square} \phi^{*}\right),
$$

but only in the space of nonlocal nonintegrated densities. As a consequence, the equation (126) cannot take place in the space of local nonintegrated densities, where the admitted deformations belong. This further yields that neither (122) can hold in this space. So, $\Gamma$ of the form (121) must be made to vanish, which happens if

$$
\bar{k}(1+k)=0, \quad k(1+k)=0 .
$$

The nontrivial solution to (129) reads as

$$
k=-1 \text {, }
$$

and $\bar{k}$ remains an arbitrary real constant. Replacing (130) in (112), and then in (118), we infer that

$$
\begin{aligned}
& b_{2}^{\text {(int) }}=0 \\
& b_{1}^{\text {(int) }}=-\frac{1}{2} \phi^{*}\left(\partial^{\mu} \phi\right) \eta^{\nu} h_{\mu \nu} .
\end{aligned}
$$

Inserting now (130) in (113), we obtain

$$
\begin{aligned}
\Lambda_{0}^{(\mathrm{int})}+2 \delta b_{1}^{(\mathrm{int})}= & \gamma\left\{-\frac{1}{8}\left(h^{2}-2 h_{\mu \nu} h^{\mu \nu}\right)\left(\partial_{\rho} \phi\right) \partial^{\rho} \phi-\right. \\
& -\left(h_{\mu \rho} h^{\rho}{ }_{\nu}-\frac{1}{2} h h_{\mu \nu}\right)\left(\partial^{\nu} \phi\right) \partial^{\mu} \phi+
\end{aligned}
$$




$$
\begin{aligned}
& +\phi^{2}\left[\frac{1}{3} \mathcal{R}_{\mu \nu} h^{\mu \nu}-\frac{1}{2} \mathcal{K} h+\frac{1}{6} \sigma^{\mu \mu_{1}} \sigma^{\nu \nu_{1}} \sigma^{\rho \rho_{1}} \times\right. \\
& \left.\times\left(\stackrel{(1)}{\Gamma}_{\mu \nu \nu_{1}} \stackrel{(1)}{\Gamma}_{\mu_{1} \rho \rho_{1}}-\stackrel{(1)}{\Gamma}_{\mu \nu \rho} \stackrel{(1)}{\Gamma} \mu_{\mu_{1} \nu_{1} \rho_{1}}\right)\right]- \\
& -\bar{k} \phi\left[\frac{2}{3} \mathcal{R}_{\mu \nu} h^{\mu \nu}-\mathcal{K} h-\frac{1}{6} \sigma^{\mu \mu_{1}} \sigma^{\nu \nu_{1}} \sigma^{\rho \rho_{1}} \times\right. \\
& \times\left(\stackrel{(1)}{\Gamma}_{\mu \nu \rho} \stackrel{(1)}{\Gamma} \mu_{1} \nu_{1} \rho_{1}-2 \stackrel{(1)}{\Gamma}_{\mu \nu \nu_{1}} \stackrel{(1)}{\Gamma}_{\mu_{1} \rho \rho_{1}}\right)+ \\
& +\left(-\frac{1}{2} \mathcal{W}^{\mu \nu \rho \lambda} \mathcal{W}_{\mu \nu \rho \lambda} h+4 \mathcal{W}^{\mu \alpha \nu \beta} \sigma^{\rho \lambda} \stackrel{(1)}{\Gamma}_{\rho \mu \nu} \stackrel{(1)}{\Gamma} \lambda \alpha \beta+\right. \\
& \left.\left.+8\left(\mathcal{W}^{\mu \alpha \nu \beta} h_{\mu \nu} \mathcal{K}_{\alpha \beta}+\frac{1}{2} \mathcal{W}^{\mu \alpha \nu \beta} \mathcal{W}_{\mu \rho \nu \beta} h_{\alpha}^{\rho}\right)\right) v(\phi)\right]- \\
& -G h-F\left(\phi^{2} \mathcal{K}+2 T^{\mu \nu} h_{\mu \nu}\right)- \\
& \left.-J(\phi)\left(\partial_{\rho} \phi\right)\left(\partial^{\rho} \phi\right)\left(\partial_{\mu} \phi\right)\left(\partial_{\nu} \phi\right)\left(\sigma^{\mu \nu} h-4 h^{\mu \nu}\right)\right\}+ \\
& +2 \bar{k}\left[\left(-\bar{k} \mathcal{K}+\frac{d F}{d \phi}\left(\partial_{\rho} \phi\right) \partial^{\rho} \phi+\frac{d G}{d \phi}\right) \xi+\right. \\
& \left.+2 F\left(\partial_{\rho} \phi\right) \partial^{\rho} \xi\right]-2\left(\frac{d G}{d \phi} \phi-4 G\right) \xi+ \\
& +\left(\partial_{\mu} F\right)\left[2 \phi^{2} \partial^{\mu} \xi-\xi \partial^{\mu}\left(\phi^{2}\right)\right]+ \\
& +\mathcal{W}^{\mu \alpha \nu \beta} \mathcal{W}_{\mu \alpha \nu \beta}(\bar{k}-\phi) \frac{d v(\phi)}{d \phi} \xi+ \\
& +2\left[\left(\partial_{\mu} \phi\right)\left(\partial^{\mu} \phi\right)\right]^{2} \xi\left[-\left(\frac{d J(\phi)}{d \phi} \phi+4 J(\phi)\right)+\bar{k} \frac{d J(\phi)}{d \phi}\right]+ \\
& +8\left(\partial_{\mu} \phi\right)\left(\partial^{\mu} \phi\right)\left(\partial_{\nu} \phi\right)\left(\partial^{\nu} \xi\right) J(\phi)(\bar{k}-\phi)+\partial_{\mu} n_{0}^{\mu} .
\end{aligned}
$$

By comparing (133) with (119), we remark that the consistency of the firstorder deformation requires that

$$
\begin{aligned}
\Theta= & 2 \bar{k}\left\{\left[-\bar{k} \mathcal{K}+\frac{d F}{d \phi}\left(\partial_{\rho} \phi\right) \partial^{\rho} \phi+\frac{d G}{d \phi}\right] \xi+2 F\left(\partial_{\rho} \phi\right) \partial^{\rho} \xi\right\}- \\
& -2\left(\frac{d G}{d \phi} \phi-4 G\right) \xi+\left(\partial_{\mu} F\right)\left[2 \phi^{2} \partial^{\mu} \xi-\xi \partial^{\mu}\left(\phi^{2}\right)\right]+ \\
& +\mathcal{W}^{\mu \alpha \nu \beta} \mathcal{W}_{\mu \alpha \nu \beta}(\bar{k}-\phi) \frac{d v(\phi)}{d \phi} \xi+
\end{aligned}
$$




$$
\begin{aligned}
& +2\left[\left(\partial_{\mu} \phi\right)\left(\partial^{\mu} \phi\right)\right]^{2} \xi\left[-\left(\frac{d J(\phi)}{d \phi} \phi+4 J(\phi)\right)+\bar{k} \frac{d J(\phi)}{d \phi}\right]+ \\
& +8\left(\partial_{\mu} \phi\right)\left(\partial^{\mu} \phi\right)\left(\partial_{\nu} \phi\right)\left(\partial^{\nu} \xi\right) J(\phi)(\bar{k}-\phi)
\end{aligned}
$$

must be of the form

$$
\Theta=\gamma \theta+\partial_{\mu} \chi^{\mu}
$$

for some local $\theta$ and $\chi^{\mu}$. Assume that (135) is satisfied. Acting with $\gamma$ on it, it follows that

$$
\gamma \Theta=\partial_{\mu}\left(\gamma \chi^{\mu}\right)
$$

Using (134), by direct computation we get that

$$
\gamma \Theta=\partial_{\mu}\left(2 \bar{k}^{2} \xi \partial^{\mu} \xi\right)
$$

By means of (136) - (137), we arrive at

$$
2 \bar{k}^{2} \xi \partial^{\mu} \xi=\gamma \chi^{\mu}+\partial_{\nu} \lambda^{\nu \mu}
$$

with $\lambda^{\nu \mu}$ some antisymmetric, but otherwise arbitrary functions

$$
\lambda^{\nu \mu}=-\lambda^{\mu \nu} .
$$

The relation (138) expresses the compatibility between the equations (136) and (137). On behalf of

$$
\xi=\gamma\left(\frac{1}{8} h\right)-\frac{1}{4} \partial^{\nu} \eta_{\nu}
$$

we determine

$$
\begin{aligned}
2 \xi \partial^{\mu} \xi= & \gamma\left[-\frac{1}{2}\left(\frac{1}{2} h \partial^{\mu} \xi+\eta_{\nu} \mathcal{K}^{\mu \nu}\right)\right]+ \\
& +\partial_{\nu}\left(-\frac{1}{4} \eta^{[\nu} \partial^{\mu]} \xi\right)+\partial_{\nu}\left(-\frac{1}{4} \eta^{(\nu} \partial^{\mu)} \xi\right)
\end{aligned}
$$

The presence of the term $\partial_{\nu}\left(-\frac{1}{4} \eta^{(\nu} \partial^{\mu)} \xi\right)$ in the right-hand side of (141) indicates that the equations (136) and (137) are compatible if

$$
\bar{k}=0 .
$$


Taking into account the formula (142), we conclude that the first-order deformation is consistent if

$$
\begin{aligned}
\Theta^{\prime}= & -2\left(\frac{d G}{d \phi} \phi-4 G\right) \xi+\left(\partial_{\mu} F\right)\left[2 \phi^{2} \partial^{\mu} \xi-\xi \partial^{\mu}\left(\phi^{2}\right)\right]- \\
& -2\left[\left(\partial_{\mu} \phi\right)\left(\partial^{\mu} \phi\right)\right]^{2}\left(\frac{d J(\phi)}{d \phi} \phi+4 J(\phi)\right) \xi- \\
& -8\left(\partial_{\mu} \phi\right)\left(\partial^{\mu} \phi\right)\left(\partial_{\nu} \phi\right)\left(\partial^{\nu} \xi\right) \phi J(\phi)- \\
& -\phi \mathcal{W}^{\mu \alpha \nu \beta} \mathcal{W}_{\mu \alpha \nu \beta} \frac{d v(\phi)}{d \phi} \xi
\end{aligned}
$$

can be written as

$$
\Theta^{\prime}=\gamma \theta^{\prime}+\partial_{\mu} \chi^{\prime \mu}
$$

for some local $\theta^{\prime}$ and $\chi^{\prime \mu}$. Using again (140), after some computation we find that $\Theta^{\prime}$ may be expressed in the form (144) if

$$
\begin{aligned}
\partial_{\mu} F & =0, \quad \frac{d v(\phi)}{d \phi}=0, \quad \frac{d G}{d \phi} \phi-4 G=\bar{C}, \\
J(\phi) & =0,
\end{aligned}
$$

with $\bar{C}$ a real constant. The equations (145) provide the solutions

$$
F(\phi)=C_{1}, \quad v(\phi)=C_{2}, \quad G(\phi)=\kappa \phi^{4}-\frac{1}{4} \bar{C},
$$

with $\kappa, C_{1}$, and $C_{2}$ some real constants. The first solution (147) is not interesting here since, in agreement with (104), it gives a term proportional with the free scalar field lagrangian at the level of the first-order deformation, which is trivial

$$
C_{1}\left(\partial_{\rho} \phi\right) \partial^{\rho} \phi=\partial_{\rho}\left(C_{1} \phi \partial^{\rho} \phi\right)+s\left(-C_{1} \phi \phi^{*}\right),
$$

so we can take

$$
F(\phi)=0 .
$$

The second solution from (147) leads, via (95), to

$$
\bar{a}_{0}^{\prime \text { (int) }}=\frac{1}{2} C_{2} \mathcal{W}_{\mu \nu \rho \lambda} \mathcal{W}^{\mu \nu \rho \lambda} .
$$


We observe that $\bar{a}_{0}^{\prime(\mathrm{int})}$ like in (150) does not describe the interaction between the scalar field and the Weyl graviton, so we can choose $C_{2}=0$ and obtain

$$
v(\phi)=0
$$

and

$$
\bar{a}_{0}^{\prime(\mathrm{int})}=0
$$

The third solution from (147) can be written as $G(\phi)=\kappa \phi^{4}-\partial_{\mu}\left(\frac{1}{16} \bar{C} x^{\mu}\right)$. Since $\bar{a}_{0}^{(\phi)}$ given in (104) is defined up to a total divergence, we can set, without loss of generality, $\bar{C}=0$, so we get

$$
G(\phi)=\kappa \phi^{4}
$$

Plugging (142), (146), (149), (151), and (153) into (133), we infer that

$$
\begin{aligned}
b_{0}^{(\mathrm{int})}= & \frac{1}{16}\left(h^{2}-2 h_{\mu \nu} h^{\mu \nu}\right)\left(\partial_{\rho} \phi\right)\left(\partial^{\rho} \phi\right)+ \\
& +\frac{1}{2}\left(h_{\mu \rho} h_{\nu}^{\rho}-\frac{1}{2} h h_{\mu \nu}\right)\left(\partial^{\mu} \phi\right)\left(\partial^{\nu} \phi\right)- \\
& -\frac{1}{2} \phi^{2}\left(\frac{1}{3} \mathcal{R}_{\mu \nu} h^{\mu \nu}-\frac{1}{2} \mathcal{K} h\right)-\frac{1}{12} \phi^{2} \sigma^{\mu \mu_{1}} \sigma^{\nu \nu_{1}} \sigma^{\rho \rho_{1}} \times \\
& \times\left(\stackrel{(1)}{\Gamma}_{\mu \nu \nu_{1}} \stackrel{(1)}{\Gamma} \mu_{\mu 1} \rho \rho_{1}-\stackrel{(1)}{\Gamma}_{\mu \nu \rho} \stackrel{(1)}{\Gamma}_{\mu_{1} \nu_{1} \rho_{1}}\right)+\frac{1}{2} \kappa h \phi^{4} .
\end{aligned}
$$

The formulas (131), (132), and (154) offer us the complete form of the interacting part from the second-order deformation of the solution to the master equation, $S_{2}^{(\mathrm{int})}$. Meanwhile, with the help of the relations (130), (142), (146), (149), (151), and (153) replaced in (105), we also gain the final form of the first-order deformation

$$
\begin{aligned}
S_{1}^{(\mathrm{int})}= & \int d^{4} x\left\{-\phi^{*}\left[\phi \xi-\left(\partial^{\mu} \phi\right) \eta_{\mu}\right]+\frac{\phi^{2}}{2} \sigma^{\mu \nu} \mathcal{K}_{\mu \nu}-\right. \\
& \left.-\frac{1}{2} h_{\mu \nu}\left[\left(\partial^{\mu} \phi\right) \partial^{\nu} \phi-\frac{1}{2} \sigma^{\mu \nu}\left(\partial^{\rho} \phi\right) \partial_{\rho} \phi\right]+\kappa \phi^{4}\right\} .
\end{aligned}
$$

In this manner, so far we have completely determined both the first- and second-order deformations. 


\section{$5 \quad$ Interacting theory}

In order to identify the interacting theory, we start from the decomposition of $g_{\mu \nu}$ like

$$
g_{\mu \nu}=\sigma_{\mu \nu}+\lambda h_{\mu \nu} .
$$

Then, the inverse of $g_{\mu \nu}$, to be denoted by $g^{\mu \nu}\left(g_{\mu \nu} g^{\nu \rho}=\delta_{\mu}{ }^{\rho}\right)$, starts like

$$
g^{\mu \nu}=\stackrel{(0)}{g}^{\mu \nu}+\lambda \stackrel{(1)}{g}^{\mu \nu}+\lambda^{2} \stackrel{(2)}{g}^{\mu \nu}+\cdots=\sigma^{\mu \nu}-\lambda h^{\mu \nu}+\lambda^{2} h^{\mu}{ }_{\rho} h^{\rho \nu}+\cdots .
$$

Based on the relations (156)-(157), we obtain that the expansions of the scalar curvature and $\sqrt{-g}$ begin like

$$
\begin{aligned}
R= & \lambda \stackrel{(1)}{R}+\lambda^{2} \stackrel{(2)}{R}+\cdots=\lambda \mathcal{R}-\lambda^{2}\left[2 h^{\mu \nu} \mathcal{R}_{\mu \nu}+\right. \\
& \left.+\sigma^{\rho \lambda} \sigma^{\mu \alpha} \sigma^{\nu \beta}\left(\stackrel{(1)}{\Gamma} \underset{\rho \mu \alpha}{\Gamma} \stackrel{(1)}{\Gamma}_{\lambda \beta \nu}-\stackrel{(1)}{\Gamma}_{\rho \mu \beta} \stackrel{(1)}{\Gamma} \lambda \alpha \nu\right)\right]+\cdots, \\
\sqrt{-g}= & \sqrt{-\operatorname{det}\left(g_{\mu \nu}\right)}=\sqrt[(0)]{-g}+\lambda \stackrel{(1)}{\sqrt{-g}}+\lambda^{2} \sqrt[(2)]{-g}+\cdots= \\
= & 1+\frac{\lambda}{2} h+\frac{\lambda^{2}}{8}\left(h^{2}-2 h_{\mu \nu} h^{\mu \nu}\right)+\cdots .
\end{aligned}
$$

The piece of antighost number zero in $S_{1}^{(\mathrm{int})}$ is nothing but the interacting lagrangian at order one in the coupling constant

$$
\mathcal{L}_{1}^{(\mathrm{int})}=a_{0}^{(\mathrm{int})}+a_{0}^{(\phi)}=\frac{1}{12} \phi^{2} \mathcal{R}-\frac{1}{2}\left(h^{\mu \nu}-\frac{1}{4} h \sigma^{\mu \nu}\right)\left(\partial_{\mu} \phi\right) \partial_{\nu} \phi+\kappa \phi^{4},
$$

which, according to (157)-(159), can be put in the form

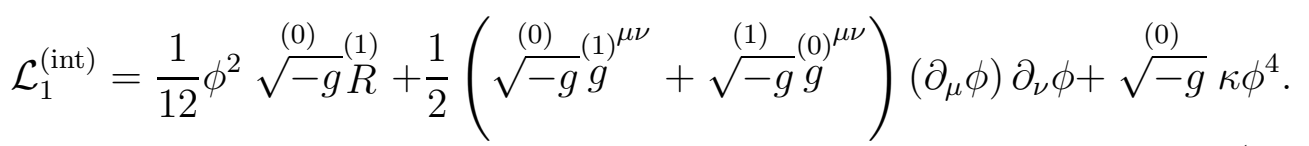

Along the same line, the term of antighost number zero in $S_{2}^{(\text {int) }}$ produces the cross-coupling lagrangian at order two as

$$
\begin{aligned}
\mathcal{L}_{2}^{(\text {int })}= & b_{0}^{(\text {int })}=\frac{1}{2}\left[h^{\mu \rho} h_{\rho}^{\nu}-\frac{1}{2} h h^{\mu \nu}+\frac{1}{8}\left(h^{2}-2 h_{\alpha \beta} h^{\alpha \beta}\right) \sigma^{\mu \nu}\right] \times \\
& \times\left(\partial_{\mu} \phi\right) \partial_{\nu} \phi+\frac{1}{12} \phi^{2}\left[\frac{1}{2} h \mathcal{R}-2 \mathcal{R}_{\mu \nu} h^{\mu \nu}-\sigma^{\mu \mu_{1}} \sigma^{\nu \nu_{1}} \sigma^{\rho \rho_{1}} \times\right. \\
& \left.\times\left(\stackrel{(1)}{\Gamma}_{\mu \nu \nu_{1}} \Gamma_{\mu_{1} \rho \rho_{1}}^{(1)}-\stackrel{(1)}{\Gamma} \mu \stackrel{(1)}{\Gamma}_{\mu \nu} \mu_{\mu_{1} \nu_{1} \rho_{1}}\right)\right]+\frac{1}{2} h \kappa \phi^{4} .
\end{aligned}
$$


Using again (157)-(159), we have that

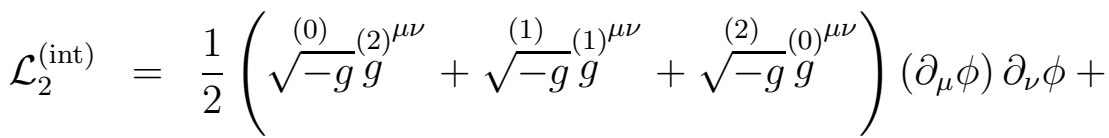

$$
\begin{aligned}
& +\frac{1}{12} \phi^{2}\left(\stackrel{(1)}{ }^{-g} \stackrel{(1)}{R}+\sqrt{-g}^{(0)} \stackrel{(2)}{R}\right)+\stackrel{(1)}{\sqrt{-g}} \kappa \phi^{4} .
\end{aligned}
$$

Taking into account (161) and (163), as well as the expansions

$$
\begin{aligned}
& \sqrt{-g} g^{\mu \nu}=\left(\sqrt{-g} g^{\mu \nu}\right)^{(0)}+\lambda\left(\sqrt{-g} g^{\mu \nu}\right)^{(1)}+\lambda^{2}\left(\sqrt{-g} g^{\mu \nu}\right)^{(2)}+\cdots=
\end{aligned}
$$

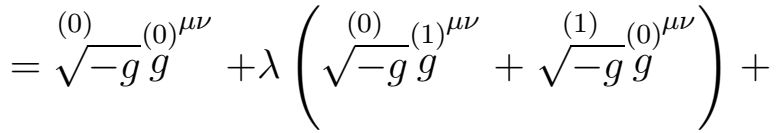

$$
\begin{aligned}
& \left.+\lambda^{2}\left({\stackrel{(0)}{\sqrt{-g}^{g}}}^{(2)^{\mu \nu}}+\stackrel{(1)}{-g}^{(1)}\right)^{\mu \nu}+\sqrt{-g}^{(2)} \stackrel{(0)}{g}^{\mu \nu}\right)+\cdots, \\
& \sqrt{-g} R=\lambda(\sqrt{-g} R)^{(1)}+\lambda^{2}(\sqrt{-g} R)^{(2)}+\cdots=
\end{aligned}
$$

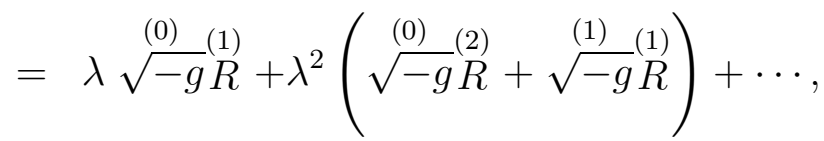

we finally find that

$$
\begin{aligned}
& \mathcal{L}_{0}^{(\phi)}+\lambda \mathcal{L}_{1}^{(\text {int })}+\lambda^{2} \mathcal{L}_{2}^{(\text {int })}+\cdots= \\
= & \left(\left(\sqrt{-g} g^{\mu \nu}\right)^{(0)}+\lambda\left(\sqrt{-g} g^{\mu \nu}\right)^{(1)}+\lambda^{2}\left(\sqrt{-g} g^{\mu \nu}\right)^{(2)}+\cdots\right) \times \\
& \times\left(\partial_{\mu} \phi\right) \partial_{\nu} \phi+\frac{1}{12} \phi^{2}\left(\lambda(\sqrt{-g} R)^{(1)}+\lambda^{2}(\sqrt{-g} R)^{(2)}+\cdots\right)+ \\
& +\lambda(\sqrt[(0)]{(\sqrt{-g}}+\lambda \sqrt{-g}+\cdots) \kappa \phi^{4}
\end{aligned}
$$

The formula (166) shows us that $\mathcal{L}_{0}^{(\phi)}+\lambda \mathcal{L}_{1}^{(\mathrm{int})}+\lambda^{2} \mathcal{L}_{2}^{(\mathrm{int})}+\cdots$ comes from the expansion of the fully deformed lagrangian

$$
\mathcal{L}^{(\mathrm{int})}=\sqrt{-g}\left\{\frac{1}{2}\left[g^{\mu \nu}\left(\partial_{\mu} \phi\right) \partial_{\nu} \phi+\frac{1}{6} \phi^{2} R\right]+\lambda \kappa \phi^{4}\right\} .
$$


The elements from the deformed solution to the master equation that are linear in the antifield of the scalar field furnish the deformed gauge transformation of this field like

$$
\begin{aligned}
\delta_{\epsilon} \phi & =\lambda\left[\left(\partial_{\mu} \phi\right) \epsilon^{\mu}-\phi \epsilon\right]-\frac{1}{2} \lambda^{2}\left(\partial_{\mu} \phi\right) \epsilon^{\nu} h_{\nu}{ }^{\mu}+\cdots= \\
& =\lambda \stackrel{(1)}{\delta_{\epsilon}} \phi+\lambda^{2} \stackrel{(2)}{\delta_{\epsilon}} \phi+\cdots .
\end{aligned}
$$

The first two orders from the above gauge transformation can be put in the form

$$
\begin{aligned}
& \left.\stackrel{(1)}{\delta_{\epsilon}} \phi=\left(\partial_{\mu} \phi\right) \stackrel{(0)}{\bar{\epsilon}}\right)^{\mu}-\phi \epsilon, \\
& \stackrel{(2)}{\delta}_{\epsilon} \phi=\left(\partial_{\mu} \phi\right) \stackrel{(1)}{\bar{\epsilon}},
\end{aligned}
$$

where

$$
\begin{aligned}
\stackrel{(0)}{\bar{\epsilon}}^{\mu} & =\epsilon^{\mu}=\epsilon^{a} \delta_{a}{ }^{\mu}, \\
\overline{(1)}^{\mu} & =-\frac{1}{2} \epsilon^{a} h_{a}{ }^{\mu} .
\end{aligned}
$$

Thus, the gauge transformation of the scalar field is expressed as

$$
\delta_{\epsilon} \phi=\lambda\left[\left(\partial_{\mu} \phi\right)\left(\overline{(0)}^{\mu}+\lambda \overline{(1)}^{\mu}+\cdots\right)-\phi \epsilon\right] .
$$

The last formula emphasizes that the gauge transformation (173) comes from the perturbative expansion of the fully deformed one

$$
\delta_{\epsilon} \phi=\lambda\left[\left(\partial_{\mu} \phi\right) \bar{\epsilon}^{\mu}-\phi \epsilon\right]
$$

where the gauge parameters $\bar{\epsilon}^{\mu}$ are given by

$$
\bar{\epsilon}^{\mu}=\left(\delta_{a}{ }^{\mu}-\frac{\lambda}{2} h_{a}{ }^{\mu}+\cdots\right) \epsilon^{a}=e_{a}{ }^{\mu} \epsilon^{a},
$$

with $e_{a}{ }^{\mu}$ the vierbein field. The conclusion of this section is that the interactions between a single Weyl graviton and a real scalar field are described by the coupled lagrangian (167), while the scalar field is endowed with the gauge symmetry (174). 


\section{Impossibility of cross-couplings between dif- ferent Weyl gravitons via a scalar field}

Now, we start from the action

$$
S_{0}^{\mathrm{L}}\left[h_{\mu \nu}^{A}, \phi\right]=\frac{1}{2} \int d^{4} x\left[\mu_{A B} \mathcal{W}_{\mu \nu \alpha \beta}^{A} \mathcal{W}^{B \mu \nu \alpha \beta}+\left(\partial_{\mu} \phi\right)\left(\partial^{\mu} \phi\right)-m^{2} \phi^{2}\right],
$$

where $\mu_{A B}$ are some constants and $\mathcal{W}_{\mu \nu \alpha \beta}^{A}$ is the linearized Weyl tensor in four spacetime dimensions corresponding to the field $h_{\mu \nu}^{A}$ from the collection, with $A=1,2, \cdots, n$. The gauge transformations of the action (176) are given by

$$
\delta_{\epsilon} h_{\mu \nu}^{A}=\partial_{(\mu} \epsilon_{\nu)}^{A}+2 \sigma_{\mu \nu} \epsilon^{A}, \quad \delta_{\epsilon} \phi=0
$$

In this section, under the hypotheses of locality, smoothness of the interactions in the coupling constant, Poincaré invariance, (background) Lorentz invariance, and the preservation of the number of derivatives on each field, together with the supplementary assumption that the internal metric $\mu_{A B}$ defined by the sum of Weyl lagrangians is positively defined, we prove that there are no indirect cross-couplings between different Weyl gravitons in the presence of a scalar field. The assumption that $\mu_{A B}$ is positively defined was employed in [3] in order to prove that there are no direct cross-couplings among different Weyl gravitons. In the case of Weyl gravity, there is no strong reason for taking the internal metric to be positively defined. However, we work under this presumption, for a proper comparison of our results to those from [3]. This assumption allows us to normalize the metric such that $\mu_{A B}=\delta_{A B}$. From now on we will use everywhere in the paper the latter form of the internal metric.

The fields/ghosts and antifields from the BRST complex are denoted by

$$
\begin{aligned}
& \Phi^{a_{0}}=\left(h_{\mu \nu}^{A}, \phi\right), \quad \eta^{a_{1}}=\left(\eta_{\mu}^{A}, \xi^{A}\right), \\
& \Phi_{a_{0}}^{*}=\left(h_{A}^{* \mu \nu}, \phi^{*}\right), \quad \eta_{a_{1}}^{*}=\left(\eta_{A}^{* \mu}, \xi_{A}^{*}\right) .
\end{aligned}
$$

The BRST differential splits like in (8), while the actions of $\delta$ and $\gamma$ on the generators from the BRST complex are given by

$$
\begin{aligned}
\delta h_{A}^{* \mu \nu} & =2 \partial_{\alpha} \partial_{\beta} \mathcal{W}_{A}^{\mu \alpha \nu \beta}, \quad \delta \phi^{* \mu}=\left(\square+m^{2}\right) \phi \\
\delta \eta_{A}^{* \mu} & =-2 \partial_{\nu} h_{A}^{* \mu \nu}, \quad \delta \xi_{A}^{*}=2 h_{A}^{*} \\
\delta \Phi^{a_{0}} & =0, \quad \delta \eta^{a_{1}}=0
\end{aligned}
$$




$$
\begin{aligned}
\gamma \Phi_{a_{0}}^{*} & =0, \quad \gamma \eta_{a_{1}}^{*}=0, \\
\gamma h_{\mu \nu}^{A} & =\partial_{(\mu} \eta_{\nu)}^{A}+2 \sigma_{\mu \nu} \xi^{A}, \quad \gamma \phi=0 \\
\gamma \eta_{\mu}^{A} & =0, \quad \gamma \xi^{A}=0 .
\end{aligned}
$$

The notation $h_{A}^{*}$ signifies the trace of $h_{A}^{* \mu \nu}, h_{A}^{*}=\sigma_{\mu \nu} h_{A}^{* \mu \nu}$. The solution to the master equation reads as

$$
\bar{S}=S_{0}^{\mathrm{L}}\left[h_{\mu \nu}^{A}, \phi\right]+\int d^{4} x h_{A}^{* \mu \nu}\left(\partial_{(\mu} \eta_{\nu)}^{A}+2 \sigma_{\mu \nu} \xi^{A}\right) .
$$

The first-order deformation of the solution to the master equation can be decomposed, like in the case of a single Weyl graviton, into

$$
\alpha=\alpha^{(\mathrm{W})}+\alpha^{(\mathrm{int})}+\alpha^{(\phi)}
$$

where $\alpha^{(\mathrm{W})}$ splits like [3]

$$
\alpha^{(\mathrm{W})}=\alpha_{2}^{(\mathrm{W})}+\alpha_{1}^{(\mathrm{W})}+\alpha_{0}^{(\mathrm{W})},
$$

with

$$
\alpha_{2}^{(\mathrm{W})}=C_{B C}^{A}\left(\eta_{A}^{* \mu}\left(\frac{1}{2} \eta^{B \nu} \partial_{[\mu} \eta_{\nu]}^{C}+\eta_{\mu}^{B} \xi^{C}\right)-\xi_{A}^{*} \eta_{\mu}^{B} \partial^{\mu} \xi^{C}\right)
$$

In formula (189) the coefficients $C_{B C}^{A}$ are some constants. The consistency of $\alpha_{2}^{(\mathrm{W})}$ in antighost number one requires the symmetry of the above coefficients in their lower indices 4

$$
C_{B C}^{A}=C_{C B}^{A}
$$

Taking into consideration the relation (190), we infer $\alpha_{1}^{(\mathrm{W})}$ in the form

$$
\alpha_{1}^{(\mathrm{W})}=\frac{1}{2} h_{A}^{* \mu \nu} C_{B C}^{A}\left[-\eta^{B \rho}\left(\partial_{(\mu} h_{\nu) \rho}^{C}-2 \partial_{\rho} h_{\mu \nu}^{C}\right)+4 h_{\mu \nu}^{B} \xi^{C}+h_{\rho(\mu}^{B} \partial_{\nu)} \eta^{C \rho}\right] .
$$

In order to obtain a consistent $\alpha_{0}^{(\mathrm{W})}$ from this $\alpha_{1}^{(\mathrm{W})}$, it follows that the constants $C_{B C}^{A}$ are further subject to the relations $[3]^{5}$

$$
C_{A B C}=\frac{1}{3} C_{(A B C)},
$$

\footnotetext{
${ }^{4}$ The piece (189) differs from that used in 3] through a $\gamma$-exact term, which does not change the condition (190).

${ }^{5}$ The piece (191) differs from that given in $[3$ through a $\delta$-exact term, which does not affect (192).
} 
where, by definition, $C_{A B C}=\delta_{A D} C_{B C}^{D}$. With (192) at hand, we find that the resulting $\alpha_{0}^{(\mathrm{W})}$ reads as in [3] (where this component is denoted by $a_{0}$ and $C_{A B C}$ by $\left.a_{a b c}\right)$.

Acting in the same manner like in subsection 4.2, we find that $\alpha^{(\mathrm{int})}=$ $\alpha_{1}^{(\text {int })}+\alpha_{0}^{(\text {int })}$, with

$$
\begin{aligned}
\alpha_{1}^{(\mathrm{int})} & =\bar{k}_{A} \phi^{*} \xi^{A}+k_{A} \phi^{*}\left[\phi \xi^{A}-\left(\partial^{\mu} \phi\right) \eta_{\mu}^{A}\right] \\
\alpha_{0}^{(\mathrm{int})}= & -\bar{k}_{A} \phi \sigma^{\mu \nu} \mathcal{K}_{\mu \nu}^{A}-\frac{1}{2} k_{A} \phi^{2} \sigma^{\mu \nu} \mathcal{K}_{\mu \nu}^{A}+ \\
& +\frac{1}{2} k_{A} h_{\mu \nu}^{A}\left[\left(\partial^{\mu} \phi\right) \partial^{\nu} \phi-\frac{1}{2} \sigma^{\mu \nu}\left(\partial^{\rho} \phi\right) \partial_{\rho} \phi\right]+ \\
& +\frac{1}{2} \mathcal{W}_{\mu \nu \rho \lambda}^{A} \mathcal{W}_{A}^{\mu \nu \rho \lambda} v(\phi),
\end{aligned}
$$

where $k_{A}$ and $\bar{k}_{A}$ are arbitrary real constants. The deformations (193)-(194) correspond to the situation where $m^{2}=0$. Meanwhile, we get in a direct way that

$$
\alpha^{(\phi)}=a_{0}^{(\phi)}
$$

where $a_{0}^{(\phi)}$ is given in (104).

Next, we investigate the consistency of the first-order deformation. In view of this, it is useful to make the following notations:

$$
\begin{aligned}
\hat{S}_{1}^{(\mathrm{W})} & =\int d^{4} x \alpha^{(\mathrm{W})} \\
\hat{S}_{1}^{(\mathrm{int})} & =\int d^{4} x\left(\alpha^{(\mathrm{int})}+\alpha^{(\phi)}\right) \\
\hat{S}_{1} & =\hat{S}_{1}^{(\mathrm{W})}+\hat{S}_{1}^{(\mathrm{int})} .
\end{aligned}
$$

By expressing the second-order deformation under the form

$$
\hat{S}_{2}=\hat{S}_{2}^{(\mathrm{W})}+\hat{S}_{2}^{(\mathrm{int})},
$$

we observe that the equation

$$
\left(\hat{S}_{1}, \hat{S}_{1}\right)+2 s \hat{S}_{2}=0
$$


controlling the second-order deformation, equivalently splits into

$$
\begin{array}{r}
\left(\hat{S}_{1}^{(\mathrm{W})}, \hat{S}_{1}^{(\mathrm{W})}\right)+2 s \hat{S}_{2}^{(\mathrm{W})}=0, \\
2\left(\hat{S}_{1}^{(\mathrm{W})}, \hat{S}_{1}^{(\mathrm{int})}\right)+\left(\hat{S}_{1}^{(\mathrm{int})}, \hat{S}_{1}^{(\mathrm{int})}\right)+2 s \hat{S}_{2}^{(\mathrm{int})}=0 .
\end{array}
$$

The equation (201) emphasizes that the constants $C_{B C}^{A}$ must satisfy the conditions [3]

$$
C_{A[B}^{D} C_{C] D}^{E}=0
$$

so they are the structure constants of a finite-dimensional, commutative, symmetric, and associative real algebra $\mathcal{A}$. As it has been shown in [14, such an algebra displays a trivial structure, in the sense that it can be written like a direct sum of some one-dimensional ideals. Consequently, we deduce that

$$
C_{B C}^{A}=0 \quad \text { if } B \neq C \text {, or } A \neq C \text {, or } A \neq B .
$$

The last formula actually implies that only the constants $C_{A A}^{A}$ may be nonvanishing.

In the sequel we analyze the equation (202). If we denote by $\hat{\Lambda}^{\text {(int) }}$ and $\beta^{\text {(int) }}$ the nonintegrated densities of the functionals $2\left(\hat{S}_{1}^{(\mathrm{W})}, \hat{S}_{1}^{(\mathrm{int})}\right)+$ $\left(\hat{S}_{1}^{(\mathrm{int})}, \hat{S}_{1}^{(\mathrm{int})}\right)$ and respectively of $\hat{S}_{2}^{(\mathrm{int})}$, the local expression of the equation (202) becomes

$$
\hat{\Lambda}^{(\mathrm{int})}=-2 s \beta^{(\mathrm{int})}+\partial_{\mu} q^{\mu}
$$

with

$$
\operatorname{gh}\left(\hat{\Lambda}^{(\mathrm{int})}\right)=1, \quad \operatorname{gh}\left(\beta^{(\mathrm{int})}\right)=0, \quad \operatorname{gh}\left(q^{\mu}\right)=1 .
$$

In our case we obtain the following decomposition of $\hat{\Lambda}^{\text {(int) }}$ with respect to the antighost number:

$$
\hat{\Lambda}^{(\text {int })}=\hat{\Lambda}_{0}^{(\mathrm{int})}+\hat{\Lambda}_{1}^{(\mathrm{int})}, \quad \operatorname{agh}\left(\hat{\Lambda}_{I}^{\text {(int) }}\right)=I, \quad I=0,1
$$

with

$$
\begin{aligned}
\hat{\Lambda}_{1}^{\text {(int })=} & -2\left(k_{A} C_{B C}^{A}+k_{B} k_{C}\right)\left[\phi^{*} \phi \eta^{B \mu} \partial_{\mu} \xi^{C}+\phi^{*}\left(\partial^{\mu} \phi\right) \eta_{\mu}^{B} \xi^{C}+\right. \\
& \left.+\frac{1}{2} \phi^{*}\left(\partial^{\mu} \phi\right) \eta^{B \nu} \partial_{[\mu} \eta_{\nu]}^{C}\right]-\left(\bar{k}_{A} k_{B}-\bar{k}_{B} k_{A}\right) \phi^{*} \xi^{A} \xi^{B}- \\
& -2\left(\bar{k}_{A} C_{B C}^{A}+k_{B} \bar{k}_{C}\right) \phi^{*} \eta_{\mu}^{B} \partial^{\mu} \xi^{C}+ \\
& +\gamma\left[k_{A} k_{B} \phi^{*}\left(\partial^{\mu} \phi\right) \eta^{A \nu} h_{\mu \nu}^{B}\right]
\end{aligned}
$$




$$
\begin{aligned}
& \hat{\Lambda}_{0}^{(\mathrm{int})}=-2\left(\bar{k}_{A} \bar{k}_{B}+k_{A} k_{B} \phi^{2}\right) \mathcal{K}^{A} \xi^{B}+2 k_{A}\left(h_{\mu \nu}^{A}-\frac{1}{2} \sigma_{\mu \nu} h^{A}\right) \times \\
& \times\left[\bar{k}_{B}\left(\partial^{\mu} \phi\right) \partial^{\nu} \xi^{B}+k_{B}\left(\left(\partial^{\mu} \phi\right) \partial^{\nu}\left(\phi \xi^{B}\right)-\left(\partial^{\mu} \phi\right) \partial^{\nu}\left(\partial^{\rho} \phi \eta_{\rho}^{B}\right)\right)\right]- \\
& -2\left(\bar{k}_{A} k_{B}+k_{A} \bar{k}_{B}\right) \phi \mathcal{K}^{A} \xi^{B}+2 k_{B}\left(\bar{k}_{A}+k_{A} \phi\right) \mathcal{K}^{A}\left(\partial^{\rho} \phi\right) \eta_{\rho}^{B}+ \\
& +k_{A} C_{B C}^{A}\left[\left(\partial^{\mu} \phi\right) \partial^{\nu} \phi-\frac{1}{2} \sigma^{\mu \nu}\left(\partial^{\rho} \phi\right) \partial_{\rho} \phi\right]\left(-\eta^{B \lambda} \partial_{\mu} h_{\nu \lambda}^{C}+\eta^{B \lambda} \partial_{\lambda} h_{\mu \nu}^{C}+\right. \\
& \left.+2 \xi^{B} h_{\mu \nu}^{C}+h_{\lambda \mu}^{B} \partial_{\nu} \eta^{C \lambda}\right)+\frac{1}{6} C_{B C}^{A}\left(2 \bar{k}_{A}+k_{A} \phi\right) \phi\left(\partial^{\mu} \partial^{\nu}-\sigma^{\mu \nu} \square\right) \times \\
& \times\left(\eta^{B \lambda} \partial_{\mu} h_{\nu \lambda}^{C}-\eta^{B \lambda} \partial_{\lambda} h_{\mu \nu}^{C}-2 \xi^{B} h_{\mu \nu}^{C}-h_{\lambda \mu}^{B} \partial_{\nu} \eta^{C \lambda}\right)+ \\
& +2 \bar{k}_{A}\left[\left(\frac{d F}{d \phi}\left(\partial_{\rho} \phi\right) \partial^{\rho} \phi+\frac{d G}{d \phi}\right) \xi^{A}+2 F(\phi)\left(\partial_{\rho} \phi\right) \partial^{\rho} \xi^{A}\right]+ \\
& +2 k_{A}\left[\left(\frac{d F}{d \phi}\left(\partial_{\rho} \phi\right) \partial^{\rho} \phi+\frac{d G}{d \phi}\right)\left(\phi \xi^{A}-\left(\partial^{\mu} \phi\right) \eta_{\mu}^{A}\right)+\right. \\
& \left.+2 F(\phi)\left(\partial_{\rho} \phi\right) \partial^{\rho}\left(\phi \xi^{A}-\left(\partial^{\mu} \phi\right) \eta_{\mu}^{A}\right)\right]+ \\
& +2\left[\left(\partial_{\mu} \phi\right)\left(\partial^{\mu} \phi\right)\right]^{2} \xi^{A}\left[k_{A}\left(\frac{d J(\phi)}{d \phi} \phi+4 J(\phi)\right)+\bar{k}_{A} \frac{d J(\phi)}{d \phi}\right]+ \\
& +8\left(\partial_{\mu} \phi\right)\left(\partial^{\mu} \phi\right)\left(\partial_{\nu} \phi\right)\left(\partial^{\nu} \xi^{A}\right) J(\phi)\left(\bar{k}_{A}+k_{A} \phi\right)+ \\
& +\gamma\left[\mathcal { W } _ { A } ^ { \mu \alpha \nu \beta } v ( \phi ) C _ { B C } ^ { A } \left(8 \mathcal{K}_{\mu \nu}^{B} h_{\alpha \beta}^{C}+4 \stackrel{(1)}{\Gamma}_{\rho \mu \nu}^{B} \stackrel{(1)}{\Gamma}_{\lambda \alpha \beta}^{C} \sigma^{\rho \lambda}+\right.\right. \\
& \left.+4 \mathcal{W}_{\mu \rho \nu \beta}^{B} h_{\alpha}^{C \rho}-\frac{1}{2} \mathcal{W}_{\mu \alpha \nu \beta}^{B} h^{C}\right)+ \\
& \left.+k_{A} J(\phi)\left(\partial_{\rho} \phi\right)\left(\partial^{\rho} \phi\right)\left(\partial_{\mu} \phi\right)\left(\partial_{\nu} \phi\right)\left(\sigma^{\mu \nu} h^{A}-4 h^{A \mu \nu}\right)\right]+ \\
& +\mathcal{W}_{A}^{\mu \alpha \nu \beta} \mathcal{W}_{\mu \alpha \nu \beta}^{A} \frac{d v(\phi)}{d \phi}\left(\bar{k}_{B}+k_{B} \phi\right) \xi^{B}- \\
& -\mathcal{W}_{A}^{\mu \alpha \nu \beta} \mathcal{W}_{\mu \alpha \nu \beta}^{B} \frac{d v(\phi)}{d \phi}\left(\partial^{\rho} \phi\right)\left(C_{B C}^{A}+\delta_{B}^{A} k_{C}\right) \eta_{\rho}^{C} .
\end{aligned}
$$

The decomposition (207) implies that $\beta^{\text {(int) }}$ and $q^{\mu}$ can be represented like

$$
\begin{aligned}
\beta^{(\mathrm{int})} & =\beta_{0}^{(\mathrm{int})}+\beta_{1}^{(\mathrm{int})}+\beta_{2}^{(\mathrm{int})}, \quad \operatorname{agh}\left(\beta_{I}^{(\mathrm{int})}\right)=I, I=0,1,2, \\
q^{\mu} & =q_{0}^{\mu}+q_{1}^{\mu}+q_{2}^{\mu}, \quad \operatorname{agh}\left(q_{I}^{\mu}\right)=I, \quad I=0,1,2 .
\end{aligned}
$$

So, by projecting the equation (205) on various antighost numbers, we find 
the tower of equations

$$
\begin{aligned}
\gamma \beta_{2}^{(\mathrm{int})} & =\partial_{\mu}\left(\frac{1}{2} q_{2}^{\mu}\right) \\
\hat{\Lambda}_{1}^{(\mathrm{int})} & =-2\left(\delta \beta_{2}^{\text {(int) }}+\gamma \beta_{1}^{\text {(int) }}\right)+\partial_{\mu} q_{1}^{\mu} \\
\hat{\Lambda}_{0}^{(\mathrm{int})} & =-2\left(\delta \beta_{1}^{\text {(int) }}+\gamma \beta_{0}^{\text {(int) }}\right)+\partial_{\mu} q_{0}^{\mu}
\end{aligned}
$$

The equation (212) can always be replaced, via some trivial redefinitions, with

$$
\gamma \beta_{2}^{(\mathrm{int})}=0
$$

We notice that $\hat{\Lambda}_{1}^{(\mathrm{int})}$ expressed by (208) can be written like in (213) if

$$
\begin{aligned}
\bar{\Gamma}= & -2\left(k_{A} C_{B C}^{A}+k_{B} k_{C}\right)\left[\phi^{*} \phi \eta^{B \mu} \partial_{\mu} \xi^{C}+\phi^{*}\left(\partial^{\mu} \phi\right) \eta_{\mu}^{B} \xi^{C}+\right. \\
& \left.+\frac{1}{2} \phi^{*}\left(\partial^{\mu} \phi\right) \eta^{B \nu} \partial_{[\mu} \eta_{\nu]}^{C}\right]-\left(\bar{k}_{A} k_{B}-\bar{k}_{B} k_{A}\right) \phi^{*} \xi^{A} \xi^{B}- \\
& -2\left(\bar{k}_{A} C_{B C}^{A}+k_{B} \bar{k}_{C}\right) \phi^{*} \eta_{\mu}^{B} \partial^{\mu} \xi^{C}
\end{aligned}
$$

takes the form

$$
\bar{\Gamma}=\delta \bar{\psi}+\gamma \bar{\Pi}+\partial_{\mu} \bar{\rho}^{\mu}
$$

for some local $\bar{\psi}, \bar{\Pi}$, and $\bar{\rho}^{\mu}$. Assuming that $\bar{\Gamma}$ reads like in (217) and applying $\delta$ on the last equation, we get

$$
\delta \bar{\Gamma}=\gamma(-\delta \bar{\Pi})+\partial_{\mu}\left(\delta \bar{\rho}^{\mu}\right)
$$

From the expression of $\bar{\Gamma}$, by direct computation, we arrive at

$$
\begin{aligned}
\delta \bar{\Gamma}= & \partial_{\mu}\left\{-\left(\bar{k}_{A} k_{B}-\bar{k}_{B} k_{A}\right) \xi^{A}\left(\xi^{B} \partial^{\mu} \phi-2 \phi \partial^{\mu} \xi^{B}\right)-\right. \\
& -2\left(\bar{k}_{A} C_{B C}^{A}+k_{B} \bar{k}_{C}\right)\left[\left(\partial^{\mu} \phi\right) \eta_{\nu}^{B} \partial^{\nu} \xi^{C}+\phi \xi^{B} \partial^{\mu} \xi^{C}-\right. \\
& \left.-\frac{1}{2} \sigma^{\mu \rho} \phi\left(\partial_{[\rho} \eta_{\nu]}^{B}\right) \partial^{\nu} \xi^{C}\right]+\left(k_{A} C_{B C}^{A}+k_{B} k_{C}\right)\left[-2 \phi\left(\partial^{\mu} \phi\right) \eta^{B \nu} \partial_{\nu} \xi^{C}+\right. \\
& +\phi^{2}\left(\frac{1}{2} \sigma^{\mu \rho}\left(\partial_{[\rho} \eta_{\lambda]}^{B}\right) \partial^{\lambda} \xi^{C}-\xi^{B} \partial^{\mu} \xi^{C}\right)+ \\
& \left.\left.+2 T^{\mu \nu} \eta_{\nu}^{B} \xi^{C}+T^{\mu \nu} \eta^{B \rho} \partial_{[\nu} \eta_{\rho]}^{C}\right]\right\}+ \\
& +\gamma\left\{2\left(\bar{k}_{A} k_{B}-\bar{k}_{B} k_{A}\right) \phi \xi^{A} \mathcal{K}^{B}+2\left(\bar{k}_{A} C_{B C}^{A}+k_{B} \bar{k}_{C}\right) \times\right.
\end{aligned}
$$




$$
\begin{aligned}
& \times\left[\phi \xi^{B} \mathcal{K}^{C}-\frac{1}{2}\left(\partial^{\mu} \phi\right) h_{\mu \nu}^{B} \partial^{\nu} \xi^{C}-\left(\partial^{\mu} \phi\right) \eta^{B \nu} \mathcal{K}_{\mu \nu}^{C}+\right. \\
& \left.+\frac{1}{2} \phi\left(\partial^{\mu} h_{\mu \nu}^{B}-\partial_{\nu} h^{B}\right) \partial^{\nu} \xi^{C}\right]+\left(k_{A} C_{B C}^{A}+k_{B} k_{C}\right) \times \\
& \times\left[\phi^{2}\left(-\xi^{B} \mathcal{K}^{C}+\frac{1}{2}\left(\partial^{\mu} h_{\mu \nu}^{B}-\partial_{\nu} h^{B}\right) \partial^{\nu} \xi^{C}\right)-\phi \partial^{\mu} \phi\left(h_{\mu \nu}^{B} \partial^{\nu} \xi^{C}+\right.\right. \\
& \left.+\eta^{B \nu} \mathcal{K}_{\mu \nu}^{C}\right)+T^{\mu \nu}\left(h_{\mu \nu}^{B} \xi^{C}-\eta^{B \rho}\left(\partial_{\mu} h_{\nu \rho}^{C}-\partial_{\rho} h_{\mu \nu}^{C}\right)\right)- \\
& \left.\left.-\frac{1}{2}\left(\partial^{\mu} \phi\right)\left(\partial^{\nu} \phi\right) \sigma^{\rho \lambda} h_{\mu \rho}^{B} \partial_{[\nu} \eta_{\lambda]}^{C}\right]\right\}
\end{aligned}
$$

Since neither of $\eta_{\mu}^{A}, \partial_{[\mu} \eta_{\nu]}^{A}, \xi^{A}$, or $\partial_{\mu} \xi^{A}$ are $\delta$-exact objects, it results that the right-hand side of (219) can be put in the form of the right-hand side of (218) if the relation (126) is fulfilled in the space of local nonintegrated densities. As it has been shown in the subsection 4.3, the relation (126) cannot hold in this space, so $\bar{\Gamma}$ must vanish

$$
\bar{\Gamma}=0 \text {. }
$$

This takes place if the constants $k_{A}$ and $\bar{k}_{A}$ are subject to the equations

$$
\begin{aligned}
\bar{k}_{A} k_{B}-\bar{k}_{B} k_{A} & =0, \\
k_{A} C_{B C}^{A}+k_{B} k_{C} & =0, \\
\bar{k}_{A} C_{B C}^{A}+k_{B} \bar{k}_{C} & =0 .
\end{aligned}
$$

Let us analyze the conditions (221)-(223). The first one, (221), exhibits two types of solutions, namely

$$
\bar{k}_{A}=q k_{A}
$$

and respectively

$$
k_{A}=p \bar{k}_{A},
$$

where $p$ and $q$ are some real numbers. Initially, we consider the solution (224). If $q \neq 0$, then the equations (223)-(224) yield

$$
\bar{k}_{A} C_{B C}^{A}+\frac{1}{q} \bar{k}_{B} \bar{k}_{C}=0
$$

The relation (204), combined with (222)-(223) and (226), ensures that

$$
k_{B} k_{C}=0, \quad k_{B} \bar{k}_{C}=0, \quad \bar{k}_{B} \bar{k}_{C}=0, \quad \text { if } B \neq C .
$$


Whenever the relations (227) hold, from (208), (213), and (220) it is easy to see that

$$
\begin{aligned}
& \beta_{2}^{\text {(int) }}=0 \\
& \beta_{1}^{\text {(int) }}=-\frac{1}{2} k_{A} k_{A} \phi^{*}\left(\partial^{\mu} \phi\right) \eta^{A \nu} h_{\mu \nu}^{A} .
\end{aligned}
$$

Plugging (204) and (227) into (209), we find by direct calculation that

$$
\begin{aligned}
& \hat{\Lambda}_{0}^{(\mathrm{int})}+2 \delta \beta_{1}^{(\mathrm{int})}=\gamma\left\{k _ { A } k _ { A } \left[\frac{1}{4}\left(\partial_{\rho} \phi\right)\left(\partial^{\rho} \phi\right)\left(h_{\mu \nu}^{A} h^{A \mu \nu}-\frac{1}{2} h^{A} h^{A}\right)-\right.\right. \\
& -\left(\partial^{\mu} \phi\right)\left(\partial^{\nu} \phi\right)\left(\sigma^{\rho \lambda} h_{\mu \rho}^{A} h_{\nu \lambda}^{A}-\frac{1}{2} h^{A} h_{\mu \nu}^{A}\right)+ \\
& +\frac{1}{3} \phi^{2}\left(\mathcal{R}_{\mu \nu}^{A} h^{A \mu \nu}-\frac{1}{4} \mathcal{R}^{A} h^{A}\right)- \\
& \left.-\frac{1}{6} \sigma^{\mu \nu} \sigma^{\rho \lambda} \sigma^{\alpha \beta} \phi^{2}\left(\stackrel{(1)}{\Gamma}_{\mu \rho \alpha}^{A} \stackrel{(1)}{\Gamma}_{\nu \lambda \beta}^{A}-\stackrel{(1)}{\Gamma}_{\mu \rho \lambda}^{A} \stackrel{(1)}{\Gamma}_{\nu \alpha \beta}^{A}\right)\right]+ \\
& +k_{A} \bar{k}_{A} \phi\left[\frac{2}{3}\left(\mathcal{R}_{\mu \nu}^{A} h^{A \mu \nu}-\frac{1}{4} \mathcal{R}^{A} h^{A}\right)-\right. \\
& \left.-\frac{1}{3} \sigma^{\mu \nu} \sigma^{\rho \lambda} \sigma^{\alpha \beta}\left(\frac{1}{2} \stackrel{(1)}{\Gamma}_{\mu \rho \alpha}^{A} \stackrel{(1)}{\Gamma}_{\nu \lambda \beta}^{A}-\stackrel{(1)}{\Gamma}_{\mu \rho \lambda}^{A} \stackrel{(1)}{\Gamma}_{\nu \alpha \beta}^{A}\right)\right]+ \\
& +C_{A A}^{A} v(\phi) \mathcal{W}_{A}^{\mu \alpha \nu \beta}\left(8 \mathcal{K}_{\mu \nu}^{A} h_{\alpha \beta}^{A}+4 \sigma^{\rho \lambda} \stackrel{(1)}{\Gamma}_{\rho \mu \nu}^{A} \stackrel{(1)}{\Gamma}_{\lambda \alpha \beta}^{A}+\right. \\
& \left.+4 \mathcal{W}_{\mu \rho \nu \beta}^{A} h_{\alpha}^{A \rho}-\frac{1}{2} \mathcal{W}_{\mu \alpha \nu \beta}^{A} h^{A}\right)+k_{A}\left[G(\phi) h^{A}-\right. \\
& -2 F(\phi)\left(\left(\partial^{\mu} \phi\right) \partial^{\nu} \phi-\frac{1}{2} \sigma^{\mu \nu}\left(\partial_{\rho} \phi\right) \partial^{\rho} \phi\right) h_{\mu \nu}^{A}+ \\
& \left.\left.+2 F(\phi) \phi^{2} \mathcal{K}^{A}\right]+k_{A} J(\phi)\left(\partial_{\rho} \phi\right)\left(\partial^{\rho} \phi\right)\left(\partial_{\mu} \phi\right)\left(\partial_{\nu} \phi\right)\left(\sigma^{\mu \nu} h^{A}-4 h^{A \mu \nu}\right)\right\}+ \\
& +2\left\{\left[-\bar{k}_{A} \bar{k}_{A} \mathcal{K}^{A}+k_{A}\left(\frac{d G(\phi)}{d \phi} \phi-4 G(\phi)\right)+\right.\right. \\
& +\left(\frac{d F(\phi)}{d \phi}\left(\partial^{\rho} \phi\right) \partial_{\rho} \phi+\frac{1}{2} \mathcal{W}_{B}^{\mu \alpha \nu \beta} \mathcal{W}_{\mu \alpha \nu \beta}^{B} \frac{d v(\phi)}{d \phi}\right)\left(\bar{k}_{A}+k_{A} \phi\right)+ \\
& \left.+\bar{k}_{A} \frac{d G(\phi)}{d \phi}\right] \xi^{A}+\left(\partial^{\rho} \phi\right)\left(2 \bar{k}_{A} F(\phi)-k_{A} \frac{d F(\phi)}{d \phi} \phi^{2}\right) \partial_{\rho} \xi^{A}-
\end{aligned}
$$




$$
\begin{aligned}
& \left.-\frac{1}{2} \mathcal{W}_{A}^{\mu \alpha \nu \beta} \mathcal{W}_{\mu \alpha \nu \beta}^{A} \frac{d v(\phi)}{d \phi}\left(\partial^{\rho} \phi\right)\left(C_{A A}^{A} \eta_{\rho}^{A}+k_{C} \eta_{\rho}^{C}\right)\right\}+ \\
& +2\left[\left(\partial_{\mu} \phi\right)\left(\partial^{\mu} \phi\right)\right]^{2} \xi^{A}\left[k_{A}\left(\frac{d J(\phi)}{d \phi} \phi+4 J(\phi)\right)+\bar{k}_{A} \frac{d J(\phi)}{d \phi}\right]+ \\
& +8\left(\partial_{\mu} \phi\right)\left(\partial^{\mu} \phi\right)\left(\partial_{\nu} \phi\right)\left(\partial^{\nu} \xi^{A}\right) J(\phi)\left(\bar{k}_{A}+k_{A} \phi\right)+\partial_{\mu} q_{0}^{\mu} .
\end{aligned}
$$

Comparing now (230) with (214), we observe that the consistency of the first-order deformation requires that

$$
\begin{aligned}
\hat{\Theta}= & 2\left\{\left[-\bar{k}_{A} \bar{k}_{A} \mathcal{K}^{A}+k_{A}\left(\frac{d G(\phi)}{d \phi} \phi-4 G(\phi)\right)+\right.\right. \\
& +\left(\frac{d F(\phi)}{d \phi}\left(\partial^{\rho} \phi\right) \partial_{\rho} \phi+\frac{1}{2} \mathcal{W}_{B}^{\mu \alpha \nu \beta} \mathcal{W}_{\mu \alpha \nu \beta}^{B} \frac{d v(\phi)}{d \phi}\right)\left(\bar{k}_{A}+k_{A} \phi\right)+ \\
& \left.+\bar{k}_{A} \frac{d G(\phi)}{d \phi}\right] \xi^{A}+\left(\partial^{\rho} \phi\right)\left(2 \bar{k}_{A} F(\phi)-k_{A} \frac{d F(\phi)}{d \phi} \phi^{2}\right) \partial_{\rho} \xi^{A}- \\
& \left.-\frac{1}{2} \mathcal{W}_{A}^{\mu \alpha \nu \beta} \mathcal{W}_{\mu \alpha \nu \beta}^{A} \frac{d v(\phi)}{d \phi}\left(\partial^{\rho} \phi\right)\left(C_{A A}^{A} \eta_{\rho}^{A}+k_{C} \eta_{\rho}^{C}\right)\right\}+ \\
& +2\left[\left(\partial_{\mu} \phi\right)\left(\partial^{\mu} \phi\right)\right]^{2} \xi^{A}\left[k_{A}\left(\frac{d J(\phi)}{d \phi} \phi+4 J(\phi)\right)+\bar{k}_{A} \frac{d J(\phi)}{d \phi}\right]+ \\
& +8\left(\partial_{\mu} \phi\right)\left(\partial^{\mu} \phi\right)\left(\partial_{\nu} \phi\right)\left(\partial^{\nu} \xi^{A}\right) J(\phi)\left(\bar{k}_{A}+k_{A} \phi\right) .
\end{aligned}
$$

must be of the form

$$
\hat{\Theta}=\gamma \hat{\theta}+\partial_{\mu} \hat{\chi}^{\mu}
$$

for some local $\hat{\theta}$ and $\hat{\chi}^{\mu}$. Assume that (232) holds. This implies the equation

$$
\gamma \hat{\Theta}=\partial_{\mu}\left(\gamma \hat{\chi}^{\mu}\right)
$$

Acting with $\gamma$ on the formula (231), we are led to

$$
\gamma \hat{\Theta}=\partial_{\mu}\left(2 \bar{k}_{A} \bar{k}_{A} \xi^{A} \partial^{\mu} \xi^{A}\right) .
$$

Comparing (233) with (234), we get that

$$
2 \bar{k}_{A} \bar{k}_{A} \xi^{A} \partial^{\mu} \xi^{A}=\gamma \hat{\chi}^{\mu}+\partial_{\nu} \hat{\lambda}^{\nu \mu},
$$

where $\hat{\lambda}^{\nu \mu}=-\hat{\lambda}^{\mu \nu}$. The relation (235) indicates that the equations (233) and (234) are compatible. On behalf of the formula (141) where we make the changes

$$
\xi \rightarrow \xi^{A}, \quad \eta_{\nu} \rightarrow \eta_{\nu}^{A}, \quad h \rightarrow h^{A}, \quad \mathcal{K} \rightarrow \mathcal{K}^{A},
$$


we find that the equations (233) $-(234)$ are compatible if

$$
\bar{k}_{A}=0 .
$$

Under these circumstances, the consistency of the first-order deformation implies that the quantity

$$
\begin{aligned}
\hat{\Theta}^{\prime}= & 2\left\{\left[k_{A}\left(\frac{d G(\phi)}{d \phi} \phi-4 G(\phi)\right)+\right.\right. \\
& \left.+\left(\frac{d F(\phi)}{d \phi}\left(\partial^{\rho} \phi\right) \partial_{\rho} \phi+\frac{1}{2} \mathcal{W}_{B}^{\mu \alpha \nu \beta} \mathcal{W}_{\mu \alpha \nu \beta}^{B} \frac{d v(\phi)}{d \phi}\right) k_{A} \phi\right] \xi^{A}+ \\
& +\left(\partial^{\rho} \phi\right)\left(2 \bar{k}_{A} F(\phi)-k_{A} \frac{d F(\phi)}{d \phi} \phi^{2}\right) \partial_{\rho} \xi^{A}- \\
& \left.-\frac{1}{2} \mathcal{W}_{A}^{\mu \alpha \nu \beta} \mathcal{W}_{\mu \alpha \nu \beta}^{A} \frac{d v(\phi)}{d \phi}\left(\partial^{\rho} \phi\right)\left(C_{A A}^{A} \eta_{\rho}^{A}+k_{C} \eta_{\rho}^{C}\right)\right\}+ \\
& +2 k_{A}\left[\left(\partial_{\mu} \phi\right)\left(\partial^{\mu} \phi\right)\right]^{2} \xi^{A}\left(\frac{d J(\phi)}{d \phi} \phi+4 J(\phi)\right)+ \\
& +8 k_{A}\left(\partial_{\mu} \phi\right)\left(\partial^{\mu} \phi\right)\left(\partial_{\nu} \phi\right)\left(\partial^{\nu} \xi^{A}\right) \phi J(\phi)
\end{aligned}
$$

must read as

$$
\hat{\Theta}^{\prime}=\gamma \hat{\theta}^{\prime}+\partial_{\mu} \hat{\chi}^{\prime \mu} .
$$

Using again (140) adapted in agreement with (236), we infer that $\hat{\Theta}^{\prime}$ given in (238) may be expressed like in (239) if the functions $J(\phi), F(\phi), v(\phi)$, and $G(\phi)$ read like in (146), (149), (151), and respectively (153). In this case it follows that

$$
\hat{\Theta}^{\prime}=0,
$$

which further yields

$$
\begin{aligned}
\beta_{0}^{(\mathrm{int})=} & -\frac{1}{2} k_{A} k_{A}\left[\frac{1}{4}\left(\partial_{\rho} \phi\right)\left(\partial^{\rho} \phi\right)\left(h_{\mu \nu}^{A} h^{A \mu \nu}-\frac{1}{2} h^{A} h^{A}\right)-\right. \\
& -\left(\partial^{\mu} \phi\right)\left(\partial^{\nu} \phi\right)\left(\sigma^{\rho \lambda} h_{\mu \rho}^{A} h_{\nu \lambda}^{A}-\frac{1}{2} h^{A} h_{\mu \nu}^{A}\right)+ \\
& +\frac{1}{3} \phi^{2}\left(\mathcal{R}_{\mu \nu}^{A} h^{A \mu \nu}-\frac{1}{4} \mathcal{R}^{A} h^{A}\right)- \\
& \left.-\frac{1}{6} \sigma^{\mu \nu} \sigma^{\rho \lambda} \sigma^{\alpha \beta} \phi^{2}\left(\stackrel{(1)}{\Gamma}_{\mu \rho \alpha}^{A} \stackrel{(1)}{\Gamma}_{\nu \lambda \beta}^{A}-\stackrel{(1)}{\Gamma}_{\mu \rho \lambda}^{A} \stackrel{(1)}{\Gamma}_{\nu \alpha \beta}^{A}\right)\right]- \\
& -\frac{1}{2} \kappa k_{A} \phi^{4} h^{A} .
\end{aligned}
$$


If $q=0$, from (224) we infer that $\bar{k}_{A}=0$, such that the interaction terms will be parametrized only by the constants $k_{A}$. Consequently, the relations (204) and (222) produce the equations

$$
k_{B} k_{C}=0 \quad \text { if } B \neq C .
$$

In this situation we have that $\beta_{2}^{\text {(int) }}$ and $\beta_{1}^{\text {(int) }}$ are still expressed by the formulas (228)-(229). By particularizing (230) to the case $\bar{k}_{A}=0$, we find that the consistency of the first-order deformation requires that $\hat{\Theta}^{\prime}$ from (238) must satisfy again the relation (239), so we obtain that $\beta_{0}^{\text {(int) }}$ still reads like in (241). The relations (228)-(229) and (241) clearly emphasize that there are no indirect couplings between different Weyl gravitons via a scalar field, irrespective of the value of $q$. Consider now the solution (225). If $p \neq 0$, from (204), (222)-(223), and (225), we obtain again the relations (227), which automatically lead to the second-order deformation given by the formulas (228) $-(229)$ and (241). In consequence, in the case $p \neq 0$ there are no indirect cross-couplings among different Weyl gravitons, so only the case $p=0$ remains to be discussed below. If $p=0$, then the equations (223) and (225) take the form

$$
\bar{k}_{A} C_{B C}^{A}=0,
$$

and respectively

$$
k_{A}=0 .
$$

In this situation, with the help of (208) and (213), we deduce that

$$
\beta_{2}^{\text {(int) }}=0, \quad \beta_{1}^{\text {(int) }}=0 .
$$

Inserting (243)-(244) in (209), we have that

$$
\begin{aligned}
\hat{\Lambda}_{0}^{(\mathrm{int})}= & \gamma\left[C _ { A A } ^ { A } v ( \phi ) \mathcal { W } _ { A } ^ { \mu \alpha \nu \beta } \left(8 \mathcal{K}_{\mu \nu}^{A} h_{\alpha \beta}^{A}+4 \sigma^{\rho \lambda} \stackrel{(1)}{\Gamma}_{\rho \mu \nu}^{A}{\stackrel{(1)}{\Gamma_{\lambda \alpha \beta}^{A}}}_{\lambda}+\right.\right. \\
& \left.\left.+4 \mathcal{W}_{\mu \rho \nu \beta}^{A} h_{\alpha}^{A \rho}-\frac{1}{2} \mathcal{W}_{\mu \alpha \nu \beta}^{A} h^{A}\right)\right]- \\
& -2 \bar{k}_{A}\left\{\left[\bar{k}_{B} \mathcal{K}^{B}-\left(\frac{d F}{d \phi}\left(\partial_{\rho} \phi\right) \partial^{\rho} \phi+\frac{d G}{d \phi}\right)\right] \xi^{A}-\right. \\
& -2 F(\phi)\left(\partial_{\rho} \phi\right) \partial^{\rho} \xi^{A}-\frac{1}{2} \mathcal{W}_{B}^{\mu \alpha \nu \beta} \mathcal{W}_{\mu \alpha \nu \beta}^{B} \frac{d v(\phi)}{d \phi} \xi^{A}+ \\
& +8\left(\partial_{\mu} \phi\right)\left(\partial^{\mu} \phi\right)\left(\partial_{\nu} \phi\right)\left(\partial^{\nu} \xi^{A}\right) J(\phi)+
\end{aligned}
$$




$$
\begin{aligned}
& \left.+2\left[\left(\partial_{\mu} \phi\right)\left(\partial^{\mu} \phi\right)\right]^{2} \xi^{A} \frac{d J(\phi)}{d \phi}\right\}- \\
& -\mathcal{W}_{A}^{\mu \alpha \nu \beta} \mathcal{W}_{\mu \alpha \nu \beta}^{A} \frac{d v(\phi)}{d \phi}\left(\partial^{\rho} \phi\right) C_{A A}^{A} \eta_{\rho}^{A}
\end{aligned}
$$

Taking into account the second relation in (245), we observe that the equation (214) requires that $\hat{\Lambda}_{0}^{\text {(int) }}$ given by (246) should be $\gamma$-exact modulo $d$

$$
\hat{\Lambda}_{0}^{(\mathrm{int})}=-2 \gamma \beta_{0}^{(\mathrm{int})}+\partial_{\mu} q_{0}^{\mu} .
$$

This takes place if

$$
\begin{aligned}
\omega= & -2 \bar{k}_{A}\left\{\left[\bar{k}_{B} \mathcal{K}^{B}-\left(\frac{d F}{d \phi}\left(\partial_{\rho} \phi\right) \partial^{\rho} \phi+\frac{d G}{d \phi}\right)\right] \xi^{A}-\right. \\
& -2 F(\phi)\left(\partial_{\rho} \phi\right) \partial^{\rho} \xi^{A}-\frac{1}{2} \mathcal{W}_{B}^{\mu \alpha \nu \beta} \mathcal{W}_{\mu \alpha \nu \beta}^{B} \frac{d v(\phi)}{d \phi} \xi^{A}+ \\
& +8\left(\partial_{\mu} \phi\right)\left(\partial^{\mu} \phi\right)\left(\partial_{\nu} \phi\right)\left(\partial^{\nu} \xi^{A}\right) J(\phi)+ \\
& \left.+2\left[\left(\partial_{\mu} \phi\right)\left(\partial^{\mu} \phi\right)\right]^{2} \xi^{A} \frac{d J(\phi)}{d \phi}\right\}- \\
& -\mathcal{W}_{A}^{\mu \alpha \nu \beta} \mathcal{W}_{\mu \alpha \nu \beta}^{A} \frac{d v(\phi)}{d \phi}\left(\partial^{\rho} \phi\right) C_{A A}^{A} \eta_{\rho}^{A},
\end{aligned}
$$

allows to be put in the form

$$
\omega=\gamma \pi+\partial_{\mu} q_{0}^{\mu}
$$

Acting with $\gamma$ on the last equation, we get that

$$
\gamma \omega=\partial_{\mu}\left(\gamma q_{0}^{\mu}\right)
$$

Applying $\gamma$ on (248), we infer that

$$
\gamma \omega=\partial_{\mu}\left(2 \bar{k}_{A} \bar{k}_{B} \xi^{A} \partial^{\mu} \xi^{B}\right) .
$$

From (250) and (251) we arrive at

$$
2 \bar{k}_{A} \bar{k}_{B} \xi^{A} \partial^{\mu} \xi^{B}=\gamma q_{0}^{\mu}+\partial_{\nu} \varkappa^{\nu \mu}
$$


with $\varkappa^{\nu \mu}=-\varkappa^{\mu \nu}$. The formula (252) states nothing but the compatibility between (250) and (251). Simple computations lead to

$$
\begin{aligned}
2 \xi^{A} \partial^{\mu} \xi^{B}= & \gamma\left[-\frac{1}{2}\left(\frac{1}{2} h^{A} \partial^{\mu} \xi^{B}+\eta_{\nu}^{A} \mathcal{K}^{B \mu \nu}\right)\right]+ \\
& +\partial_{\nu}\left(-\frac{1}{4} \eta^{A[\nu} \partial^{\mu]} \xi^{B}\right)+\partial_{\nu}\left(-\frac{1}{4} \eta^{A(\nu} \partial^{\mu)} \xi^{B}\right) .
\end{aligned}
$$

The presence of the term $\partial_{\nu}\left(-\frac{1}{4} \eta^{A(\nu} \partial^{\mu)} \xi^{B}\right)$ in the right-hand side of (253) indicates that the equations (250) and (251) are compatible if

$$
\bar{k}_{A}=0 \text {. }
$$

Inserting (254) in (248), we find that $\hat{\Lambda}_{0}^{(\text {int) }}$ can be expressed like in (247) if the quantity

$$
\omega^{\prime}=-\mathcal{W}_{A}^{\mu \alpha \nu \beta} \mathcal{W}_{\mu \alpha \nu \beta}^{A} \frac{d v(\phi)}{d \phi}\left(\partial^{\rho} \phi\right) C_{A A}^{A} \eta_{\rho}^{A}
$$

is of the form

$$
\omega^{\prime}=\gamma \pi^{\prime}+\partial_{\mu} q_{0}^{\mu}
$$

It is clear that $\omega^{\prime}$ given in (255) cannot be written like in (256), so $\omega^{\prime}$ must vanish. Its vanishing then implies that the function $v(\phi)$ must be constant. Reprising the same arguments like in the subsection 4.3, we can take $v(\phi)$ to vanish and thus (246) and (254) produce

$$
\beta_{0}^{(\mathrm{int})}=0 .
$$

Consequently, in the case $p=0$ we have that

$$
\begin{aligned}
\hat{S}_{1}^{(\mathrm{int})}= & \int d^{4} x\left(J(\phi)\left(\partial_{\mu} \phi\right)\left(\partial^{\mu} \phi\right)\left(\partial_{\rho} \phi\right) \partial^{\rho} \phi+\right. \\
& \left.+F(\phi)\left(\partial_{\rho} \phi\right) \partial^{\rho} \phi+G(\phi)\right),
\end{aligned}
$$

and

$$
\hat{S}_{2}^{(\mathrm{int})}=\cdots=\hat{S}_{k}^{(\mathrm{int})}=\cdots=0,
$$

where $J(\phi), F(\phi)$ and $G(\phi)$ are now arbitrary functions of the undifferentiated scalar field. It is simple to see that the term $\hat{S}_{1}^{(\mathrm{int})}$ given in (258) does not describe interactions between a scalar field and Weyl gravitons. Thus, the case $p=0$ corresponds to the more restrictive situation where there are no interactions at all between the Weyl gravitons and the scalar field, and the same holds for the indirect cross-couplings among different Weyl gravitons.

In conclusion, the result announced in the beginning of this section has been completely proved. 


\section{Conclusion}

To conclude with, in this paper we have investigated the indirect couplings between a collection of Weyl gravitons (described in the free limit by a sum of linearized Weyl actions) in the presence of a scalar field by using the powerful setting based on local BRST cohomology. Initially, we have obtained the couplings between a single Weyl graviton and a scalar field, and then we have proved, under the hypotheses of locality, smoothness of the interactions in the coupling constant, Poincaré invariance, (background) Lorentz invariance, and the preservation of the number of derivatives on each field, together with the supplementary assumption that the internal metric defined by the sum of Weyl lagrangians is positively defined, that there are no consistent crossinteractions among different Weyl gravitons in the presence of a scalar field.

\section{Acknowledgment}

Two of the authors (C.B. and E.M.C.) are partially supported by the European Commission FP6 program MRTN-CT-2004-005104 and by the type A grant 305/2004 with the Romanian National Council for Academic Scientific Research (C.N.C.S.I.S.) and the Romanian Ministry of Education and Research (M.E.C.). One of the authors (A.C.L.) was supported by the World Federation of Scientists (WFS) National Scholarship Programme.

\section{A Solution to the equation $\gamma_{0} \bar{a}_{0}^{\prime \prime(\text { int })}=\partial_{\mu} \bar{m}_{0}^{(\mathrm{int}) \mu}$}

In order to solve the equation

$$
\gamma_{0} \bar{a}_{0}^{\prime \prime(\mathrm{int})}=\partial_{\mu} \bar{m}_{0}^{(\mathrm{int}) \mu}
$$

we start from the requirement that $\bar{a}_{0}^{\prime \prime \text { (int) }}$ may contain at most four derivatives. Then, $\bar{a}_{0}^{\prime \prime(\mathrm{int})}$ can be decomposed like

$$
\bar{a}_{0}^{\prime \prime(\mathrm{int})}=\omega_{0}+\omega_{1}+\omega_{2}+\omega_{3}+\omega_{4},
$$

where $\left(\omega_{i}\right)_{i=\overline{0,4}}$ contains $i$ derivatives. Since there is no Lorentz scalar that can be constructed out of the first-order derivatives of the fields $h_{\mu \nu}$ and $\phi$, it results that

$$
\omega_{1}=0, \omega_{3}=0
$$


Due to the different number of derivatives in the components $\omega_{0}, \omega_{2}$, and $\omega_{4}$, the equation (260) leads to three independent equations

$$
\begin{aligned}
\gamma_{0} \omega_{0} & =\partial_{\mu} j_{0}^{\mu}, \\
\gamma_{0} \omega_{2} & =\partial_{\mu} j_{2}^{\mu}, \\
\gamma_{0} \omega_{4} & =\partial_{\mu} j_{4}^{\mu} .
\end{aligned}
$$

Since $\omega_{0}$ is derivative-free, it can be represented as

$$
\omega_{0}\left(\phi, h_{\mu \nu}\right)=f_{1}(\phi) f_{2}\left(h_{\mu \nu}\right) .
$$

Inserting (266) in (263), we obtain that

$$
f_{1}(\phi) \frac{\partial f_{2}\left(h_{\mu \nu}\right)}{\partial h_{\mu \nu}} \partial_{(\mu} \eta_{\nu)}=\partial_{\mu} j_{0}^{\mu}
$$

The left-hand side of the above relation can be written like a full divergence if

$$
\partial_{\mu}\left(f_{1}(\phi) \frac{\partial f_{2}\left(h_{\mu \nu}\right)}{\partial h_{\mu \nu}}\right)=0,
$$

which implies

$$
f_{1}(\phi) \frac{\partial f_{2}\left(h_{\mu \nu}\right)}{\partial h_{\mu \nu}}=c^{\mu \nu}
$$

with $c^{\mu \nu}$ some arbitrary, symmetric constants. The only constants with this property are

$$
c^{\mu \nu}=c \sigma^{\mu \nu},
$$

with $c$ an arbitrary, real constant. Accordingly, the equation (269) leads to

$$
f_{1}(\phi)=c^{\prime}, \quad f_{2}\left(h_{\mu \nu}\right)=c^{\prime \prime} \sigma^{\mu \nu} h_{\mu \nu},
$$

with $c^{\prime}$ and $c^{\prime \prime}$ two constants related by $c=c^{\prime} c^{\prime \prime}$. The solution (271) provides no interactions between the scalar field and the Weyl graviton, but merely reduces to the cosmological term

$$
\omega_{0}\left(\phi, h_{\mu \nu}\right)=c \sigma^{\mu \nu} h_{\mu \nu}
$$

so we can set $c=0$, and thus $\omega_{0}\left(\phi, h_{\mu \nu}\right)=0$. Let us analyze now the equation (264). If we use the notation

$$
D^{\mu \nu}=\frac{\delta \omega_{2}}{\delta h_{\mu \nu}},
$$


then we get that

$$
\gamma_{0} \omega_{2}=-2\left(\partial_{\mu} D^{\mu \nu}\right) \eta_{\nu}+\partial_{\mu} u^{\mu}
$$

with $u^{\mu}$ a local current. The relation (274) expresses the fact that $\omega_{2}$ is solution to (264) if

$$
\partial_{\mu} D^{\mu \nu}=0
$$

The solution to the last equation reads as

$$
D^{\mu \nu}=\partial_{\alpha} \partial_{\beta} U^{\mu \alpha \nu \beta}
$$

where $U^{\mu \alpha \nu \beta}$ displays the symmetry properties of the Riemann tensor and involves only the undifferentiated fields $\phi$ and $h_{\mu \nu}$.

Let $N$ be a derivation in the algebra of the fields $h_{\mu \nu}$ and of their derivatives that counts the powers of the fields and their derivatives, defined by

$$
N=\sum_{k \geq 0}\left(\partial_{\mu_{1} \cdots \mu_{k}} h_{\mu \nu}\right) \frac{\partial}{\partial\left(\partial_{\mu_{1} \cdots \mu_{k}} h_{\mu \nu}\right)} .
$$

Then, it is easy to see that for every nonintegrated density $\chi$, we have that

$$
N \chi=h_{\mu \nu} \frac{\delta \chi}{\delta h_{\mu \nu}}+\partial_{\mu} s^{\mu},
$$

where $\delta \chi / \delta h_{\mu \nu}$ denotes the variational derivative of $\chi$. If $\chi^{(l)}$ is a homogeneous polynomial of order $l>0$ in the fields and their derivatives, then

$$
N \chi^{(l)}=l \chi^{(l)}
$$

Using (273), (276), and (278), we find that

$$
N \omega_{2}=-\frac{1}{2} \mathcal{R}_{\mu \alpha \nu \beta} U^{\mu \alpha \nu \beta}+\partial_{\mu} v^{\mu}
$$

We expand $\omega_{2}$ like

$$
\omega_{2}=\sum_{l>0} \omega_{2}^{(l)}
$$

where $N \omega_{2}^{(l)}=l \omega_{2}^{(l)}$, such that

$$
N \omega_{2}=\sum_{l>0} l \omega_{2}^{(l)}
$$


Comparing (280) with (282), we reach the conclusion that the decomposition (281) induces a similar decomposition with respect to $U^{\mu \alpha \nu \beta}$, i.e.

$$
U^{\mu \alpha \nu \beta}=\sum_{l>0} U_{(l-1)}^{\mu \alpha \nu \beta}
$$

Substituting (283) into (280) and comparing the resulting expression with (282), we obtain that

$$
\omega_{2}^{(l)}=-\frac{1}{2 l} \mathcal{R}_{\mu \alpha \nu \beta} U_{(l-1)}^{\mu \alpha \nu \beta}+\partial_{\mu} \bar{v}_{(l)}^{\mu} .
$$

Introducing (284) in (281), we arrive at

$$
\omega_{2}=-\frac{1}{2} \mathcal{R}_{\mu \alpha \nu \beta} \bar{U}^{\mu \alpha \nu \beta}+\partial_{\mu} \bar{v}^{\mu}
$$

where

$$
\bar{U}^{\mu \alpha \nu \beta}=\sum_{l>0} \frac{1}{l} U_{(l-1)}^{\mu \alpha \nu \beta} .
$$

On behalf of (285), we find that

$$
\gamma_{0} \omega_{2}=\eta_{\lambda} \partial_{\rho}\left(\mathcal{R}_{\mu \alpha \nu \beta} \frac{\partial \bar{U}^{\mu \alpha \nu \beta}}{\partial h_{\rho \lambda}}\right)+\partial_{\mu} j^{\mu} .
$$

The last relation shows that $\omega_{2}$ satisfies the equation (264) if

$$
\partial_{\rho}\left(\mathcal{R}_{\mu \alpha \nu \beta} \frac{\partial \bar{U}^{\mu \alpha \nu \beta}}{\partial h_{\rho \lambda}}\right)=0 .
$$

Taking into account the fact that $\mathcal{R}_{\mu \alpha \nu \beta} \partial \bar{U}^{\mu \alpha \nu \beta} / \partial h_{\rho \lambda}$ is symmetric with respect to the indices $\rho$ and $\lambda$ and second-order in the derivatives, we obtain that

$$
\mathcal{R}_{\mu \alpha \nu \beta} \frac{\partial \bar{U}^{\mu \alpha \nu \beta}}{\partial h_{\rho \lambda}}=\partial_{\gamma} \partial_{\delta} V^{\rho \gamma \lambda \delta},
$$

where the functions $V^{\rho \gamma \lambda \delta}$ exhibit the symmetry properties of the Riemann tensor and depend only on the undifferentiated fields $\phi$ and $h_{\mu \nu}$. By computing the left-hand side of (289), we arrive at

$$
\mathcal{R}_{\mu \alpha \nu \beta} \frac{\partial \bar{U}^{\mu \alpha \nu \beta}}{\partial h_{\rho \lambda}}=\partial_{\gamma} \partial_{\delta}\left(2 h_{\alpha \nu} \frac{\partial \bar{U}^{\gamma \alpha \nu \delta}}{\partial h_{\rho \lambda}}\right)-\frac{1}{2} \frac{\partial^{2} \bar{U}^{\mu \alpha \nu \beta}}{\partial h_{\rho \lambda} \partial h_{\gamma \delta}} \times
$$




$$
\begin{aligned}
& \times\left(\partial_{[\nu} h_{\beta][\mu} \partial_{\alpha]} h_{\gamma \delta}+\partial_{[\mu} h_{\alpha][\nu} \partial_{\beta]} h_{\gamma \delta}-\right. \\
& \left.-h_{\nu[\mu} \partial_{\alpha]} \partial_{\beta} h_{\gamma \delta}+h_{\beta[\mu} \partial_{\alpha]} \partial_{\nu} h_{\gamma \delta}\right)- \\
& -\frac{1}{2} \frac{\partial^{2} \bar{U}^{\mu \alpha \nu \beta}}{\partial h_{\rho \lambda} \partial \phi}\left(\partial_{[\nu} h_{\beta][\mu} \partial_{\alpha]} \phi+\partial_{[\mu} h_{\alpha][\nu} \partial_{\beta]} \phi-\right. \\
& \left.-h_{\nu[\mu} \partial_{\alpha]} \partial_{\beta} \phi+h_{\beta[\mu} \partial_{\alpha]} \partial_{\nu} \phi\right)- \\
& -\frac{1}{2} \frac{\partial^{3} \bar{U}^{\mu \alpha \nu \beta}}{\partial h_{\rho \lambda} \partial h_{\gamma \delta} \partial h_{\varepsilon \omega}} h_{\gamma \delta,[\nu} h_{\beta][\mu} \partial_{\alpha]} h_{\varepsilon \omega}- \\
& -\frac{1}{2} \frac{\partial^{3} \bar{U}^{\mu \alpha \nu \beta}}{\partial h_{\rho \lambda} \partial \phi \partial \phi} \phi_{,[\nu} h_{\beta][\mu} \partial_{\alpha]} \phi-\frac{1}{2} \frac{\partial^{3} \bar{U}^{\mu \alpha \nu \beta}}{\partial h_{\rho \lambda} \partial h_{\gamma \delta} \partial \phi} \times \\
& \times\left(h_{\gamma \delta,[\nu} h_{\beta][\mu} \partial_{\alpha]} \phi+\phi_{,[\nu} h_{\beta][\mu} \partial_{\alpha]} h_{\gamma \delta}\right),
\end{aligned}
$$

where we made the notations

$$
\phi_{, \nu}=\partial_{\nu} \phi, \quad h_{\gamma \delta, \nu}=\partial_{\nu} h_{\gamma \delta}
$$

The right-hand side of (290) can be expressed like in the right-hand side of (289) if

$$
\frac{\partial^{2} \bar{U}^{\mu \alpha \nu \beta}}{\partial h_{\gamma \delta} \partial h_{\rho \lambda}}=0, \quad \frac{\partial^{2} \bar{U}^{\mu \alpha \nu \beta}}{\partial \phi \partial h_{\rho \lambda}}=0,
$$

whose general solution reads as

$$
\bar{U}^{\mu \alpha \nu \beta}=-\sigma^{\nu[\mu} \sigma^{\alpha] \beta} f(\phi)+\tilde{c}\left(\sigma^{\nu[\mu} h^{\alpha] \beta}-\sigma^{\beta[\mu} h^{\alpha] \nu}-\sigma^{\nu[\mu} \sigma^{\alpha] \beta} h\right),
$$

with $f(\phi)$ an arbitrary, smooth function of the undifferentiated scalar field and $\tilde{c}$ an arbitrary, real constant. Substituting (293) in (285), we deduce that, up to a total derivative, $\omega_{2}$ can be written like

$$
\omega_{2}=\mathcal{R} f(\phi)-2 \tilde{c}\left(\mathcal{R}_{\mu \nu}-\frac{1}{2} \sigma_{\mu \nu} \mathcal{R}\right) h^{\mu \nu} .
$$

We remark that the terms proportional with $\tilde{c}$ from (294) are not interacting terms, and therefore we will omit them by taking $\tilde{c}=0$, such that

$$
\omega_{2}=\mathcal{R} f(\phi) .
$$

In the end we analyze the equation (265). By means of the notation

$$
E^{\mu \nu}=\frac{\delta \omega_{4}}{\delta h_{\mu \nu}}
$$


we find that

$$
\gamma_{0} \omega_{4}=-2\left(\partial_{\mu} E^{\mu \nu}\right) \eta_{\nu}+\partial_{\mu} \bar{u}^{\mu},
$$

with $\bar{u}^{\mu}$ a local current. With the help of (297), we observe that $\omega_{4}$ satisfies the equation (265) if

$$
\partial_{\mu} E^{\mu \nu}=0 .
$$

The solution to the last equation reads as

$$
E^{\mu \nu}=\partial_{\alpha} \partial_{\beta} H^{\mu \alpha \nu \beta},
$$

where $H^{\mu \alpha \nu \beta}$ depends on $\phi$ and $h_{\mu \nu}$, exhibits the symmetries of the Riemann tensor, and contains just two derivatives. Acting similarly to $\omega_{2}$, we arrive at

$$
\omega_{4}=-\frac{1}{2} \mathcal{R}_{\mu \alpha \nu \beta} \tilde{H}^{\mu \alpha \nu \beta}+\partial_{\mu} \tilde{u}^{\mu},
$$

where

$$
\tilde{H}^{\mu \alpha \nu \beta}=\sum_{l>0} \frac{1}{l} H_{(l-1)}^{\mu \alpha \nu \beta} .
$$

It is clear that $\tilde{H}^{\mu \alpha \nu \beta}$ presents the symmetry of the Riemann tensor and includes only two derivatives. In order to preserve the differential order of the scalar field equation, it results that $\tilde{H}^{\mu \alpha \nu \beta}$ must be of the form

$$
\tilde{H}^{\mu \alpha \nu \beta}=\bar{f}(\phi) \bar{H}^{\mu \alpha \nu \beta},
$$

where $\bar{H}^{\mu \alpha \nu \beta}$ depends only on $h_{\mu \nu}$, has the symmetry properties of the Riemann tensor, and has precisely two derivatives. Substituting (302) into (300), we deduce that

$$
\omega_{4}=-\frac{1}{2} \bar{f}(\phi) \mathcal{R}_{\mu \alpha \nu \beta} \bar{H}^{\mu \alpha \nu \beta}+\partial_{\mu} \tilde{u}^{\mu} .
$$

Acting with $\gamma_{0}$ on (303), we obtain

$$
\begin{aligned}
\gamma_{0} \omega_{4}= & \eta^{\rho} \partial^{\tau}\left[\bar{f}(\phi) \mathcal{R}_{\mu \alpha \nu \beta} \frac{\partial \bar{H}^{\mu \alpha \nu \beta}}{\partial h^{\tau \rho}}-\right. \\
& -\partial^{\lambda}\left(\bar{f}(\phi) \mathcal{R}_{\mu \alpha \nu \beta} \frac{\partial \bar{H}^{\mu \alpha \nu \beta}}{\partial\left(\partial^{\lambda} h^{\tau \rho}\right)}\right)+ \\
& \left.+\partial^{\lambda} \partial^{\sigma}\left(\bar{f}(\phi) \mathcal{R}_{\mu \alpha \nu \beta} \frac{\partial \bar{H}^{\mu \alpha \nu \beta}}{\partial\left(\partial^{\lambda} \partial^{\sigma} h^{\tau \rho}\right)}\right)\right]+\partial_{\mu} \bar{j}^{\mu} .
\end{aligned}
$$


The last formula shows that $\omega_{4}$ given in (303) satisfies the equation (265) if

$$
\begin{aligned}
& \partial^{\tau}\left[\bar{f}(\phi) \mathcal{R}_{\mu \alpha \nu \beta} \frac{\partial \bar{H}^{\mu \alpha \nu \beta}}{\partial h^{\tau \rho}}-\partial^{\lambda}\left(\bar{f}(\phi) \mathcal{R}_{\mu \alpha \nu \beta} \frac{\partial \bar{H}^{\mu \alpha \nu \beta}}{\partial\left(\partial^{\lambda} h^{\tau \rho}\right)}\right)+\right. \\
& \left.+\partial^{\lambda} \partial^{\sigma}\left(\bar{f}(\phi) \mathcal{R}_{\mu \alpha \nu \beta} \frac{\partial \bar{H}^{\mu \alpha \nu \beta}}{\partial\left(\partial^{\lambda} \partial^{\sigma} h^{\tau \rho}\right)}\right)\right]=0 .
\end{aligned}
$$

The equation (305) further leads to

$$
\begin{aligned}
& \bar{f}(\phi) \mathcal{R}_{\mu \alpha \nu \beta} \frac{\partial \bar{H}^{\mu \alpha \nu \beta}}{\partial h^{\tau \rho}}-\partial^{\lambda}\left(\bar{f}(\phi) \mathcal{R}_{\mu \alpha \nu \beta} \frac{\partial \bar{H}^{\mu \alpha \nu \beta}}{\partial\left(\partial^{\lambda} h^{\tau \rho}\right)}\right)+ \\
& +\partial^{\lambda} \partial^{\sigma}\left(\bar{f}(\phi) \mathcal{R}_{\mu \alpha \nu \beta} \frac{\partial \bar{H}^{\mu \alpha \nu \beta}}{\partial\left(\partial^{\lambda} \partial^{\sigma} h^{\tau \rho}\right)}\right)=\partial^{\lambda} \partial^{\sigma} \bar{V}_{\lambda \tau \sigma \rho},
\end{aligned}
$$

where $\bar{V}_{\lambda \tau \sigma \rho}$ depends on $\phi$ and $h_{\mu \nu}$, presents the symmetry of $\mathcal{R}_{\lambda \tau \sigma \rho}$, and has two derivatives. On the other hand, the most general form of $\bar{H}^{\mu \alpha \nu \beta}$ can be represented like

$$
\begin{aligned}
\bar{H}^{\mu \alpha \nu \beta}= & a^{\gamma \delta \delta^{\prime} \xi \chi \chi^{\prime} \mu \alpha \nu \beta}\left(h_{\mu \nu}\right)\left(\partial_{\gamma} h_{\delta \delta^{\prime}}\right) \partial_{\xi} h_{\chi \chi^{\prime}}+ \\
& +b^{\gamma \delta \xi \xi^{\prime} \mu \alpha \nu \beta}\left(h_{\mu \nu}\right) \partial_{\gamma} \partial_{\delta} h_{\xi \xi^{\prime}}
\end{aligned}
$$

where the functions $a^{\gamma \delta \delta^{\prime} \xi \chi \chi^{\prime} \mu \alpha \nu \beta}\left(h_{\mu \nu}\right)$ and $b^{\gamma \delta \xi \xi^{\prime} \mu \alpha \nu \beta}\left(h_{\mu \nu}\right)$ have appropriate symmetry properties. Inserting (307) in (306), we infer that the left-hand side of the latter reads as $\partial^{\lambda} \partial^{\sigma} \bar{V}_{\lambda \tau \sigma \rho}$ if

$$
\begin{aligned}
a^{\gamma \delta \delta^{\prime} \xi \chi \chi^{\prime} \mu \alpha \nu \beta}\left(h_{\mu \nu}\right) & =0, \\
b^{\gamma \delta \xi \xi^{\prime} \mu \alpha \nu \beta}\left(h_{\mu \nu}\right) & =C^{\gamma \delta \xi \xi^{\prime} \mu \alpha \nu \beta},
\end{aligned}
$$

where $C^{\gamma \delta \xi \xi^{\prime} \mu \alpha \nu \beta}$ are some constants, and, moreover, the quantity $\frac{\partial \bar{H}^{\mu \alpha \nu \beta}}{\partial\left(\partial^{\lambda} \partial^{\sigma} h^{\tau \rho}\right)}$ inherits the symmetries of $\mathcal{R}_{\lambda \tau \sigma \rho}$. Based on the last considerations, it is simple to see that

$$
\begin{aligned}
\bar{H}^{\mu \alpha \nu \beta}= & \bar{c}_{1} \mathcal{R}^{\mu \alpha \nu \beta}+\bar{c}_{2}\left(\sigma^{\mu \nu} \mathcal{R}^{\alpha \beta}+\sigma^{\alpha \beta} \mathcal{R}^{\mu \nu}-\sigma^{\alpha \nu} \mathcal{R}^{\mu \beta}-\sigma^{\mu \beta} \mathcal{R}^{\alpha \nu}\right)+ \\
& +\bar{c}_{3}\left(\sigma^{\mu \beta} \sigma^{\alpha \nu}-\sigma^{\mu \nu} \sigma^{\alpha \beta}\right) \mathcal{R}
\end{aligned}
$$

where $\bar{c}_{1}, \bar{c}_{2}$, and $\bar{c}_{3}$ are some real constants. Replacing (310) in (303), we get, up to a total derivative, that $\omega_{4}$ takes the form

$$
\omega_{4}=\left(c_{1} \mathcal{R}_{\mu \alpha \nu \beta} \mathcal{R}^{\mu \alpha \nu \beta}+c_{2} \mathcal{R}_{\mu \nu} \mathcal{R}^{\mu \nu}+c_{3} \mathcal{R}^{2}\right) \bar{f}(\phi)
$$


with $c_{1}=-\frac{1}{2} \bar{c}_{1}, c_{2}=-2 \bar{c}_{2}$, and $c_{3}=\bar{c}_{3}$. By employing (295) and (311), we then find that the solution to the equation (260) becomes

$$
\bar{a}_{0}^{\prime \prime \text { (int) }}=\left(c_{1} \mathcal{R}_{\mu \alpha \nu \beta} \mathcal{R}^{\mu \alpha \nu \beta}+c_{2} \mathcal{R}_{\mu \nu} \mathcal{R}^{\mu \nu}+c_{3} \mathcal{R}^{2}\right) \bar{f}(\phi)+\mathcal{R} f(\phi),
$$

as stated in the subsection 4.2,

\section{References}

[1] E. S. Fradkin, A. A. Tseytlin, Conformal Supergravity, Phys. Rept. 119 (1985) 233.

[2] V. Balasubramanian, E. Gimon, D. Minic, J. Rahmfeld, Four Dimensional Conformal Supergravity From AdS Space, Phys. Rev. D63 (2001) 104009 [hep-th/0007211].

[3] N. Boulanger, M. Henneaux, A derivation of Weyl gravity, Annalen Phys. 10 (2001) 935 hep-th/0106065.

[4] C. Becchi, A. Rouet, R. Stora, Renormalization of the abelian HiggsKibble model, Commun. Math. Phys. 42 (1975) 127.

[5] I. V. Tyutin, Gauge invariance in field theory and statistical physics in operator formalism, FIAN ( LEBEDEV Physical Institute) preprint No. 39, Moscow, 1975 (in Russian), unpublished.

[6] I. A. Batalin, G. A. Vilkovisky, Gauge algebra and quantization, Phys. Lett. B102 (1981) 27.

[7] I. A. Batalin, G. A. Vilkovisky, Quantization of gauge theories with linearly dependent generators, Phys. Rev. D28 (1983) 2567; Erratumibid. D30 (1984) 508.

[8] I. A. Batalin, G. A. Vilkovisky, Existence theorem for gauge algebra, J. Math. Phys. 26 (1985) 172.

[9] M. Henneaux, Lectures on the antifield-BRST formalism for gauge theories, Nucl. Phys. B (Proc. Suppl.) 18A (1990) 47-106.

[10] M. Henneaux, C. Teitelboim, Quantization of Gauge Systems (Princeton University Press, Princeton 1992). 
[11] G. Barnich, M. Henneaux, Consistent couplings between fields with a gauge freedom and deformations of the master equation, Phys. Lett. B311 (1993) 123 hep-th/9304057.

[12] G. Barnich, F. Brandt, M. Henneaux, Local BRST cohomology in the antifield formalism. 1. General theorems, Commun. Math. Phys. 174 (1995) 57 [hep-th/9405109]; Local BRST cohomology in gauge theories, Phys. Rept. 338 (2000) 439 [hep-th/0002245].

[13] A. Van Proeyen, Superconformal tensor calculus in $N=1$ and $N=$ 2 supergravity, in "Supersymmetry and Supergravity", XIX Karpacz Winter School on Theoretical Physics, 1983, ed. B. Milewski, World Scientific Publ., Singapore (1983) 93

[14] N. Boulanger, T. Damour, L. Gualtieri, M. Henneaux, Inconsistency of interacting, multi-graviton theories, Nucl. Phys. B597 (2001) 127-171 [hep-th/0007220].

[15] N. Boulanger, M. Esole, A note on the uniqueness of $D=4, N=1 \mathrm{su}-$ pergravity, Class. Quantum Grav. 19 (2002) 2107-2124 [gr-qc/0110072].

[16] M. Henneaux, Space-time locality of the BRST formalism, Commun. Math. Phys. 140 (1991) 1. 